DOI: $10.15421 / 4220009$

УДК 519.6, 625.7/.8 (075.8)

А. В. Трофимов, канд. фриз.-мат. наук

\title{
АНАЛИЗ РЕШЕНИЯ ОБРАТНЫХ ЗАДАЧ ОЦЕНКИ СТРУКТУРНЫХ И ФУНКЦИОНАЛЬНЫХ ХАРАКТЕРИСТИК ДОРОЖНЫХ ПОКРЫТИЙ
}

Предложен метод оценки механических и геометрических параметров лростых оснований на основе решения обратных задач для многослойных упругих пакетов с использованием информационно-вероятностного (байесовского) подхода. Суть метода заключается в блочно-параметрическом приближении априорной плотности вероятности и функции правдоподобия в пространстве параметров и модельных данных задачи.

Ключевые слова: дорожные покрытия; оценка структурных параметров; обратные задачи; вероятностный подход.

Постановка проблемы. Вопросы неразрушающего мониторинга и контроля механических и геометрических параметров дорожных покрытий являются одними из важнейших в практике эксплуатации сети автомобильных дорог, взлётно-посадочных полос аэродромов и других подобных элементов транспортной инфраструктуры. Основными исходными данными, поставляемые современными приборами для расчёта таких параметров, служат:

- механические перемещения точек поверхности испытуемого покрытия под действием заданной нагрузки (например, колёс автомобиля);

- величины отражённых слоями покрытия приникающих излучений, создаваемых соответствующими источниками.

Главными характеристиками качества для подобных измерительных инструментов служат точность, возможность оценки как можно большего количества параметров тестируемого покрытия, высокая скорость получения соответствующих данных.

Приборы первого типа, позволяющие оценить степень деформации испытуемой поверхности, называются дефрлектометрами. К приборам второго типа относятся, в первую очередь, георадары.

Современные дефлектометры разделяются на два класса [12]. Приборы первого класса позволяют оценивать перемещения точек испытуемой поверхности. Их можно условно назвать «дефлектометрами перемещения». Такие инструменты используют, как правило, оптические датчики-дальномеры [5], закреплённые на днище кузова специального транспортного средства, создающего необходимое нагружение исследуемого покрытия (рис. 1). 
Разумеется, подобные инструменты должны быть оснащены также датчиком-интегратором скорости транспортного средства для точного определения промежутков времени между фиксацией результатов двух соседних датчиков перемещения в одной и той же точке исследуемой поверхности. Исключение составляют «дефлектометры падающего веca» (Falling Weight Deflectometer, FWD), которые определяют время прибытия волны возмущения и размах соответствующих колебаний точки поверхности исследуемого покрытия, вызванных падением с определённой высоты массивной металлической плиты [14]. Для определения указанных параметров колебаний используют сейсмометры и геофоны.

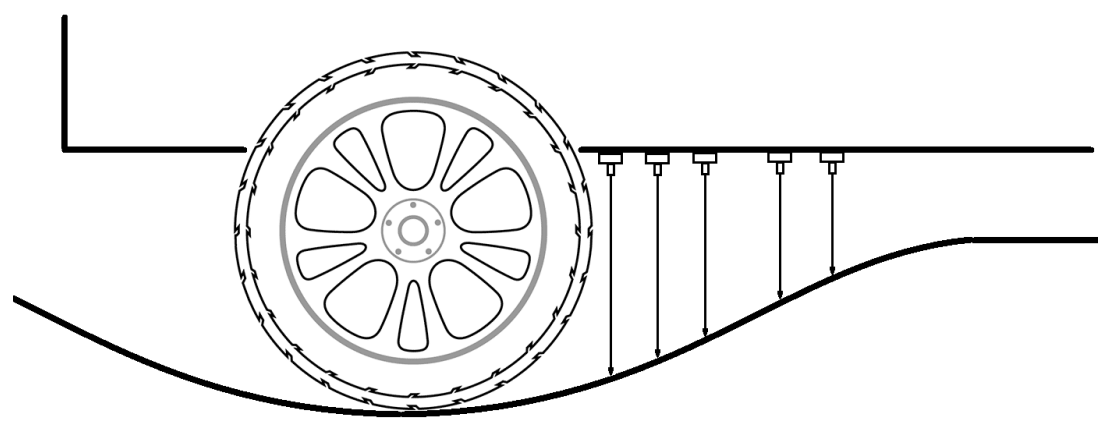

Рис. 1 - Схема расположения сканирующих датчиков в современных высокоскоростных дефлектометрах

Дефрлектометры второго типа, называемые «дефлектометрами скорости» [7], используют оптические допплеровские датчики для определения скоростей определённых точек исследуемой поверхности, которые и служат исходными данными для расчёта параметров дорожного покрытия.

Одной из основных технических проблем, возникающих при разработке динамических (созданных на базе движущегося с высокой скоростью транспортного средства) дефлектометров обоих типов, является вибрация основы крепления оптических датчиков, амплитуда которой может значительно (иногда на несколько порядков) превышать величины измеряемых перемещений. Частично эта проблема решается установкой демпфирующих устройств, а также акселерометровинтеграторов, учитывающих вертикальные и горизонтальные движения основы датчиков. В общем случае параметры таких движений должны быть включены в число неизвестных, подлежащих нахождению в процессе обработки данных измерений.

Георадары, как правило, бесполезны для непосредственного определения механических параметров исследуемого покрытия, но могут использоваться для получения геометрических характеристик многослойного основания, образующего данное покрытие (например, фрормы 
границ раздела слоёв), а также некоторых физических характеристик (например, влагосодержания), которые прямо или косвенно могут влиять на прочность рассматриваемой конструкции.

На современном этапе развития тестирующих устройств динамические дефлектометры рассмотренных типов оснащены количеством сканирующих датчиков, исчисляющихся единицами [12], что явно недостаточно для получения сколько-нибудь детальной информации о параметрах испытуемого многослойного основания. Как правило, использование подобных устройств заключается в нахождении по результатам измерений с помощью некоторых эмпирических формул определённого интегрального показателя (например, SNP, Adjusted Structural Number, [11]), характеризующего остаточную прочность испытуемой конструкции. Для более детальной оценки параметров покрытия используются исследования на основе FWD-инструментов, которые, однако, имеют низкую производительность.

В то же время существующее развитие аппаратных средств измерений, а также прогресс в развитии эффективных методов обработки данных, допускает использование большего количества исходных данных (причём данных как датчиков дефлектометра, так и георадара и иных устройств), что, очевидно, позволит оценить большее число параметров слоистого основания, и, возможно, с большей точностью. Это позволит создавать высокоскоростные инструменты мониторинга дорожных покрытий нового поколения с онлайн-интерпретацией результатов сканирования.

Сделаем обзор литературы, касающейся вопросов решения обратных задач для многослойных оснований. Большинство работ посвящено определению упругих модулей слоёв основания [15, 21], а также упругого модуля реакции грунтового основания [10]. Реже встречаются работы, посвященные определению переменных толщин слоёв основания и коэффрициента Пуассона материала слоёв [16]. Практически отсутствуют работы, в которых рассматриваются важные вопросы определения параметров нелинейности материала слоёв, а также параметров взаимодействия слоёв между собой и с упругим основанием.

В качестве физической модели для решения «прямой задачи» чаще всего рассматривают однородные упругие изотропные слои одинаковой толщины и даже модели однородного упругого полупространства с «приведёнными» толщинами слоёв [14]. Хотя использование подобных моделей основано на известных аналитических решениях (в частности, на решении Бусинесска для однородного полупространства), они всё же имеют известные ограничения, не позволяющие рассматривать, в частности, неоднородные среды, слои переменной толщины и т.п. Модели, основанные на использовании конечноэлементных решений, применяются значительно реже [6].

Большое количество работ, появившихся в последние годы, связано с использованием современных инструментов аппроксимации функций многих переменных, в том числе нейронных сетей [8, 15]. Такие модели позволяют осуществлять оперативный анализ получаемых дефлекто- 
метрами данных, однако они требуют для реализации процедур «обучения с учителем» эффрективных методов решения обратных задач для многослойных оснований с разнообразными параметрами слоёв на основе «содержательных» моделей.

Таким образом, в целом имеющийся материал по решению «обратных задач» для слоистых оснований носит разрозненный характер; отсутствует общий подход к решению таких задач, сформулированных на основе широкого класса механических моделей, позволяющий проверять обоснованность априорных данных о параметрах слоистого основания и точности измерительной аппаратуры, исключать аномальные значения наблюдаемых данных («выбросы»), а также протестировать модель с целью избежания её чрезмерной параметризации («переобучения»).

Целью данной работы является попытка развития указанных методов на базе информационно-вероятностного («байесовского») подхода, показавшего свою эффрективность при решении широкого круга задач математической фиизики (автору известна лишь одна работа, посвящённая применению этого подхода к решению обратных задач для слоистых оснований [9]; большинство же работ использует традиционный детерминистский («тихоновский») подход).

Модели слоистых оснований. Обработка данных, поставляемых рассматриваемыми инструментами, основывается на определённых моделях слоистых оснований, рассмотренных далее.

Параметры, включаемые в рассмотрение в процессе моделирования слоистых оснований, подразделяются на такие основные группы (рис. 2).

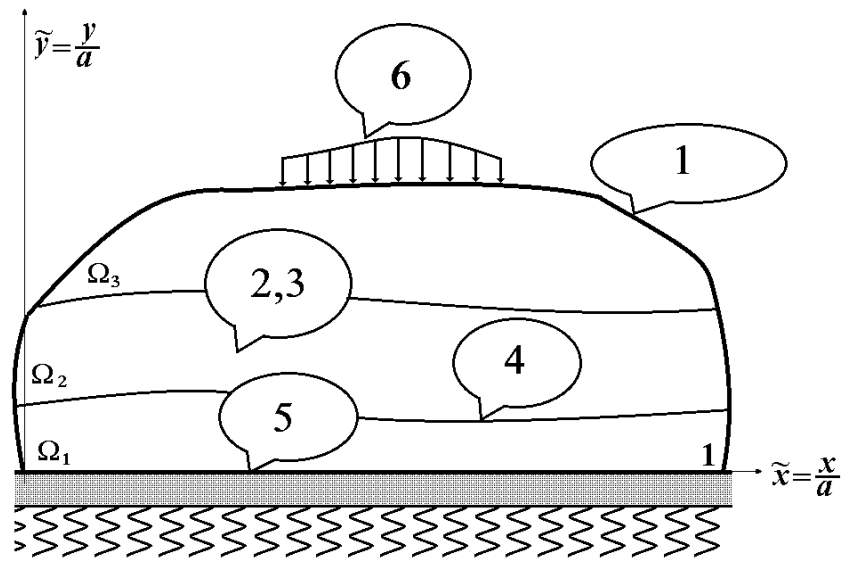

Рис. 2 - Схема слоистого пакета

1. Геометрические характеристики слоистого пакета. Эти параметры характеризуют количество и толщины слоёв, а также уравнения границ раздела слоёв и верхней свободной поверхности, определяе- 
мые обычно с помощью сплайнов или других аппроксимирующих функций.

2. Механические свойства слоёв пакета. Это, в первую очередь, упругие постоянные (например, модуль сдвига G и коэффрициент Пуассона $v$ ), а также параметры, характеризующие пластические, вязкоупругие и т.п. свойства материала слоя, а также степень развития усталостных разрушений.

3. Иные фризические параметры материала слоёв, такие как степень уплотнения, влагосодержания, пористости, вязкости и т.д.

4. Параметры интерфейса между слоями. Тип взаимодействия (жёсткое сцепление или возможность проскальзывания слоёв в соответствии, например, с законом Амонтона-Кулона и т.п.) и его параметры.

5. Характеристики грунтового основания, на котором расположен рассматриваемый пакет. На рис. 2 представлена модель основания Пастернака, характеризующегося двумя параметрами: модулем сдвига $\mathrm{G}_{\mathrm{s}}$ и коэффициентом жёсткости «пружины» k, хотя могут быть использованы модели с меньшим (основание Винклера) или большим (основание Керра) количеством параметров [14].

6. Параметры распределённой нагрузки, действию которой подвергается слоистый пакет в процессе испытаний. Характеризуют сплайнили иную приближающую функцию q.

Модель слоистого основания системы с распределёнными параметрами $\left(\boldsymbol{m}=\left(m_{1}, m_{2}, \ldots\right)^{T}\right.$ - вектор параметров), сформулированная в перемещениях, может быть выражена операторным уравнением

$$
\boldsymbol{A}[\boldsymbol{m}](\boldsymbol{u})=\boldsymbol{f}[\boldsymbol{m}] .
$$

Здесь $\boldsymbol{u}=\boldsymbol{u}(\boldsymbol{x})$ - вектор-фрункция перемещений точек пакета, определённая в составной области $\Omega=\Omega_{1} \cup \Omega_{2} \cup \ldots \cup \Omega_{\mathrm{K}} \quad\left(\boldsymbol{\Omega}_{l}, \quad i=\overline{\mathbf{1}, K}-\right.$ область, занимаемая $i$-м слоем пакета), $\boldsymbol{f}[\boldsymbol{m}]$ - вектор объёмных сил, $\boldsymbol{A}[\boldsymbol{m}](\cdot): \boldsymbol{H}_{\boldsymbol{1}}[\boldsymbol{m}] \rightarrow \boldsymbol{H}_{2}-$ векторный оператор, компоненты которого (как обычно в тензорном анализе, подразумевается суммирование по повторяющимся индексам) определяются по формуле

$$
\boldsymbol{A}_{i}[\boldsymbol{m}](\boldsymbol{u})=\frac{\partial \sigma_{i j}[\boldsymbol{m}]\left(\varepsilon_{k l}(\boldsymbol{u})\right)}{\partial x_{j}}, i, j, k=\overline{1,3} .
$$

Компоненты тензора напряжений $\sigma$ и малых деформаций $\varepsilon$ связаны определяющими соотношениями

$$
\sigma_{i j}=\sigma_{i j}[\boldsymbol{m}]\left(\varepsilon_{k l}\right) ;
$$


в свою очередь, компоненты тензора малых деформаций выражаются через компоненты вектора перемещения $\boldsymbol{u}$ соотношениями Коши Грина

$$
\varepsilon_{k l}(\boldsymbol{u})=\frac{1}{2}\left(\frac{\partial u_{k}}{\partial x_{l}}+\frac{\partial u_{l}}{\partial x_{k}}\right)
$$

Структура пространства $\boldsymbol{H}_{\boldsymbol{1}}[\boldsymbol{m}]$ векторов перемещений $\boldsymbol{u}=\boldsymbol{u}(\boldsymbol{x})$ определяется системой ограничений:

- граничных условий

$$
\begin{gathered}
\boldsymbol{\sigma}_{n}(\boldsymbol{x})=\boldsymbol{T}(\boldsymbol{x})=\left(T_{1}(\boldsymbol{x}), T_{2}(\boldsymbol{x}), T_{3}(\boldsymbol{x})\right)^{T} ; \boldsymbol{x} \in S_{\sigma}, i=1,2,3 ; \\
\boldsymbol{u}=\boldsymbol{u}_{u}(\boldsymbol{x}), \boldsymbol{x} \in S_{u}, \quad S=\partial \Omega=S_{\sigma} \cup S_{u}
\end{gathered}
$$

где $\boldsymbol{n}$ - внешняя нормаль к поверхности пакета; $\sigma_{n i}=\sigma_{i j} n_{j}$ - координаты нормальной компоненты вектора напряжений) и условий взаимодействия между слоями пакета и с грунтовым основанием.

В случае жёстко сцепленных (“склеенных") слоёв $i$ и $k$ на поверхности их контакта $S_{i k}=\partial \Omega_{i} \cap \partial \Omega_{k}$

$$
\boldsymbol{u}^{(i)}(\boldsymbol{x})=\boldsymbol{u}^{(k)}(\boldsymbol{x}), \boldsymbol{x} \in S_{i k} \text {; }
$$

- в случае контакта с проскальзыванием шероховатых слоёв в соответствии с моделью трения Амонтона - Кулона

$$
\left\{\begin{array}{l}
\boldsymbol{u}_{n}^{(i)}(\boldsymbol{x})=\boldsymbol{u}_{n}^{(k)}(\boldsymbol{x}), \\
\sigma_{\tau}^{(i)}(\boldsymbol{x})=\mu_{i k}(\boldsymbol{x}) \sigma_{n}^{(k)}(\boldsymbol{x}),
\end{array} \quad \boldsymbol{x} \in S_{i k},\right.
$$

где $\boldsymbol{n}$ - вектор нормали к поверхности контакта $S_{i k} ; \boldsymbol{\tau} \perp \boldsymbol{n}-$ единичный касательный вектор, определяемый как

$$
\begin{gathered}
\tau(\boldsymbol{x})=\frac{\boldsymbol{u}_{\tau}^{(k)}(\boldsymbol{x})-\boldsymbol{u}_{\tau}^{(i)}(\boldsymbol{x})}{\left|\boldsymbol{u}_{\tau}^{(k)}(\boldsymbol{x})-\boldsymbol{u}_{\tau}^{(i)}(\boldsymbol{x})\right|}, \boldsymbol{u}_{\tau}^{(i)}(\boldsymbol{x})=\boldsymbol{u}^{(i)}(\boldsymbol{x})-\boldsymbol{u}_{n}^{(i)}(\boldsymbol{x}), \\
\boldsymbol{u}_{\tau}^{(k)}(\boldsymbol{x})=\boldsymbol{u}^{(k)}(\boldsymbol{x})-\boldsymbol{u}_{n}^{(k)}(\boldsymbol{x}), \boldsymbol{x} \in S_{i k},
\end{gathered}
$$

Здесь $\mu_{i k}=\mu_{i k}(\boldsymbol{x})$ - коэффицциент трения между слоями $i$ и $k$.

Модель Винклера взаимодействия пакета с грунтовым основанием описывается соотношениями (трением между $K$-м слоем пакета и основанием пренебрегаем)

$$
\left\{\begin{array}{l}
\sigma_{n}^{(K)}(\boldsymbol{x})=k_{\boldsymbol{S}}(\boldsymbol{x}) u_{n}^{(K)}(\boldsymbol{x}), \\
\sigma_{\tau}^{(K)}(\boldsymbol{x})=\mu_{i k}(\boldsymbol{x}) \sigma_{n}^{(K)}(\boldsymbol{x}),
\end{array} \quad \boldsymbol{x} \in S_{K},\right.
$$


где $S_{K}$ - поверхность контакта $K$-го слоя пакета и грунтового основания; $k_{S}(\boldsymbol{x})$ - коэффициент локальной жёсткости основания.

В случае модели основания Пастернака вместо первого из соотношений (9) будем иметь

$$
\sigma_{n}^{(K)}(\boldsymbol{x})=k_{\boldsymbol{S}}(\boldsymbol{x}) u_{n}^{(K)}(\boldsymbol{x})+G_{\boldsymbol{S}}(\boldsymbol{x}) \nabla_{\zeta}^{2} u_{n}^{(K)}, \quad \boldsymbol{x} \in S_{K},
$$

где $G_{\boldsymbol{S}}(\boldsymbol{x})$ - локальный модуль сдвига основания; $\xi:\left(\xi_{1}, \xi_{2}\right)$ - естественные криволинейные (локальные) координаты, определённые на поверхности контакта $S_{K} ; \nabla_{\zeta}^{2}=\frac{\partial^{2}}{\partial \xi_{1}^{2}}+\frac{\partial^{2}}{\partial \xi_{2}^{2}}-$ оператор Лапласа в указанных координатах.

В случае модели слоистого основания, сформулированной в скоростях (в квазистатической постановке), компоненты вектора граничной нагрузки $\boldsymbol{T}$ (первое из соотношений (5)) считаются зависящими от координат $\boldsymbol{x}$ и времени $t$ :

$$
\begin{gathered}
\sigma_{n}(\boldsymbol{x}, t)=T(\boldsymbol{x}, t)=\left(T_{1}(\boldsymbol{x}, t), T_{2}(\boldsymbol{x}, t), T_{3}(\boldsymbol{x}, t)\right)^{T}, \\
\boldsymbol{x} \in S_{\sigma}, t \in\left[0, t_{\max }\right], i=1,2,3,
\end{gathered}
$$

остальные соотношения (1) - (10) записываются в скоростях перемещений $\boldsymbol{v}(\boldsymbol{x}, t)=\dot{\boldsymbol{u}}(\boldsymbol{x}, t)$ и напряжений $\dot{\sigma}_{i j}(\boldsymbol{x}, t)$. Система соотношений дополняется начальными условиями

$$
\boldsymbol{u}(\boldsymbol{x}, 0)=\boldsymbol{u}_{\boldsymbol{0}}(\boldsymbol{x}), \boldsymbol{x} \in \Omega \text {. }
$$

В обоих случаях формулировок моделей слоистого основания в состав вектора параметров $\boldsymbol{m}$ входят дискретизованные "версии" функций, характеризующих дифференциальный оператор (2) и вектор правых частей $\boldsymbol{f}$ уравнения (1) (например, входящие в определяющие соотношения (3) упругие характеристики $\boldsymbol{G}$ и $v$ слоёв пакета, а также величины, характеризующие их пластические и реологические свойства), расчётную область $\Omega$ (например, уравнения границ раздела слоёв $S_{i k}$ и границы раздела $S_{K}$ пакета и грунтового основания) и систему ограничений, определяющих структуру функционального пространства $H_{1}$ (вектор поверхностной нагрузки $\boldsymbol{T}$; коэффициент трения между слоями $\mu_{i k}$; характеристики грунтового основания $k_{\boldsymbol{S}}$ и $G_{\boldsymbol{S}}$ ).

Уравнение (1) характеризует так называемую «прямую задачу»: по данному вектору параметров $\boldsymbol{m}$ определить вектор перемещений $\boldsymbol{u}$

$$
\boldsymbol{u}=\mathfrak{R}(\boldsymbol{m}),
$$


где $\mathfrak{R}(\cdot): \boldsymbol{H}_{\boldsymbol{M}} \rightarrow \boldsymbol{H}_{\boldsymbol{1}}[\boldsymbol{m}]$ - оператор «прямой задачи»; $\boldsymbol{H}_{\boldsymbol{M}}$ - пространство изменения вектора параметров $\boldsymbol{m}$.

Для формулировки «обратной задачи» вводят в рассмотрение оператор $\aleph(\cdot): \boldsymbol{H}_{\boldsymbol{1}}[\boldsymbol{m}] \rightarrow \boldsymbol{H}_{\boldsymbol{D}}$, который для каждого $\boldsymbol{u}$ определяет так называемый «вектор модельных данных» $\boldsymbol{d}=\left(d_{1}, d_{2}, \ldots\right)^{T}$ задачи

$$
d=\aleph(u(m))=g(m),
$$

где $\boldsymbol{H}_{\boldsymbol{D}}$ - пространство модельных данных; $\boldsymbol{g}(\cdot): \boldsymbol{H}_{\boldsymbol{M}} \rightarrow \boldsymbol{H}_{\boldsymbol{D}}$.

В качестве модельных данных следует, в первую очередь, рассматривать радиальные перемещения $\boldsymbol{u}_{i}$ (или, соответственно, радиальные скорости $v_{i}$ ) определённых точек поверхности пакета $A_{i}$, сканируемые каждым из используемых дефлектометров (ис. 2)

$$
d_{i}=\sum_{j=1}^{3} l_{i j} u_{i j}, d_{i}=\left(\sum_{j=1}^{3} l_{i j} v_{i j}\right), \quad i=1,2, \ldots, K_{d},
$$

где $l_{i j}, j=1,2,3,-$ направляющие косинусы сканирующего луча $i$-го деслектометра; $K_{d}$ - общее число используемых дефлектометров. Другим примером модельных данных может служить измеряемое дальномером расстояние от места его размещения до точки свободной поверхности пакета, определяющее, по сути, уравнение части границы $\partial \Omega_{K}$.

Суть обратной задачи состоит в «обращении» оператора $g(\cdot)$ : для данного (возможно, «зашумлённого») наблюдаемого вектора данных $\boldsymbol{d}_{\text {obs }}$ найти соответствующее значение вектора параметров $\boldsymbol{m} \in \boldsymbol{H}_{\boldsymbol{M}}$. Сложность заключается в том, что отображение $\boldsymbol{g}(\cdot)$ не обязано быть гомеоморфизмом из $\boldsymbol{H}_{\boldsymbol{M}}$ в $\boldsymbol{H}_{\boldsymbol{D}}$. Нарушение условий гомеоморфности приводит к следующим ситуациям, не позволяющим напрямую разрешить обратную задачу.

1. Оператор $\boldsymbol{g}(\cdot)$ не является сюръекцией, т.е. $\boldsymbol{g}\left(\boldsymbol{H}_{\boldsymbol{M}}\right) \neq \boldsymbol{H}_{\boldsymbol{D}}$. Это означает, что для некоторого вектора $\boldsymbol{d}_{\text {obs }} \in \boldsymbol{H}_{\boldsymbol{D}}$ соответствующий ему вектор $\boldsymbol{m} \in \boldsymbol{H}_{\boldsymbol{M}}$ может не существовать.

2. Для некоторого вектора $\boldsymbol{d} \in \boldsymbol{H}_{\boldsymbol{D}}$ возможно существование как минимум двух векторов $\boldsymbol{m}_{\boldsymbol{I}} \in \boldsymbol{H}_{\boldsymbol{M}}, \boldsymbol{m}_{\boldsymbol{1}} \in \boldsymbol{H}_{\boldsymbol{M}}$, для которых $\boldsymbol{g}\left(\boldsymbol{m}_{1}\right)=\boldsymbol{g}\left(\boldsymbol{m}_{2}\right)=\boldsymbol{d}$ (нарушение единственности решения обратной задачи).

3. Для «зашумлённого» $\boldsymbol{d}_{o b s}$ и «точного» $\boldsymbol{d}_{\text {true }}$ вектора данных 


$$
\boldsymbol{d}_{\text {obs }}=\boldsymbol{d}_{\text {true }}+\boldsymbol{\varepsilon},
$$

соответствующие решения обратной задачи могут сильно различаться даже для малых значений компонент «шума» $\varepsilon$ (нарушение непрерывности решения обратной задачи).

Для преодоления указанных трудностей, связанных с нарушением гомеоморфности отображения $\boldsymbol{g}(\cdot)$, были предложены два основных подхода. Исторически первый из них, называемый «детерминистским», восходит к 60-м годам XX века [20]. Согласно этому подходу, иногда называемому «методом регуляризации А.Н. Тихонова», приближённое «обращение» оператора (11) выполняется как решение оптимизационной задачи минимизации функционала

$$
I(\boldsymbol{m})=R\left(\boldsymbol{g}(\boldsymbol{m})-\boldsymbol{d}_{\text {obs }}\right)+\alpha(\boldsymbol{\varepsilon}) \Omega(\boldsymbol{m}) \rightarrow \min , \boldsymbol{m} \in \boldsymbol{H}_{\boldsymbol{M}} .
$$

Функционал $R(\cdot)$, называемый «невязочным» или «подгоночным», играет роль проектора $\boldsymbol{H}_{\boldsymbol{D}} \rightarrow \boldsymbol{g}\left(\boldsymbol{H}_{\boldsymbol{M}}\right)$, преодолевая, таким образом, первое из указанных затруднений, связанное с существованием решения обратной задачи. Для преодоления второго и третьего затруднений (нарушение единственности решения обратной задачи и её непрерывности) в соотношении (12) вводится второй функционал $\Omega(\boldsymbol{m})$, называемый «регуляризирующим» или «стабилизирующим». Этот функционал имеет характер штрафного, накладывая дополнительные ограничения на множество возможных значений $\boldsymbol{H}_{\boldsymbol{M}}$ вектора параметров $\boldsymbol{m}$. С одной стороны, такое сужение множества изменения $\boldsymbol{m}$ может определяться некоторой априорной информацией о решении обратной задачи, что уменьшает вероятность существования нескольких минимумов фрунционала $I(\boldsymbol{m})$ (так называемой «мультимодальности»). С другой стороны, введение второго слагаемого в выражении (12) может помочь в преодолении нарушения взаимной непрерывности отображения $\boldsymbol{g}(\cdot)$. Дело в том, что в случае «сглаживающего» оператора $\boldsymbol{g}(\cdot)$ в большей степени подавляются высокочастотные компоненты вектора $\boldsymbol{m}$. Соответственно, «обратный» оператор $\boldsymbol{d} \rightarrow \boldsymbol{m}$ будет «огрубляющим», в значительной мере увеличивающим высокочастотные составляющие ошибки $\varepsilon$ вектора $\boldsymbol{d}_{\text {obs }}$. Роль функционала $\Omega(\boldsymbol{m})$ как раз и состоит в «срезании» этих высокочастотных компонент. Уровень такого «срезания» регулируется множителем $\alpha(\varepsilon)$ в зависимости от величины ошибки $\varepsilon$. Указанная идея и называется «регуляризацией» функционала невязки $R(\cdot)$.

Несмотря на удовлетворительные практические результаты, многие исследователи признавали некоторую искусственность построений типа (12). Вследствие этого в 70-80-х годах XX ст. предпринимались попытки поставить решение обратных задач на более надёжную методологическую основу. Подобные попытки, в частности, в работах [4], [18], 
привели к разработке нового подхода, названного «информационновероятностным» или «байесовским». Основу этого подхода составляют следующие положения.

1. Наиболее общим способом определения (возможной) связи между параметрами $\boldsymbol{m}$ и данными $\boldsymbol{d}$ является состояние информации, описываемое распределением вероятностей $\boldsymbol{P ( \cdot )}$ в пространстве $\boldsymbol{H}_{\boldsymbol{M}} \times \boldsymbol{H}_{\boldsymbol{D}}$, В частности (совместной) плотностью распределения $\phi(\boldsymbol{m}, \boldsymbol{d})$.

2. В качестве исходных данных для решения обратной задачи выступают два основных состояния информации. Во-первых, это теоретическая плотность распределения $\Theta(\boldsymbol{m}, \boldsymbol{d})$, выражающая фризический закон связи между $\boldsymbol{m}$ и $\boldsymbol{d}$ и учитывающая неопределённость этой связи ввиду, например, недостаточной параметризации или приближённого характера рассматриваемого закона. Во-вторых, это некоторая независимо определённая априорная плотность распределения $\rho(\boldsymbol{m}, \boldsymbol{d})$, выражающая, например, результаты ранее проведённых исследований.

3. Решением обратной задачи является апостериорная плотность распределения $\sigma(\boldsymbol{m}, \boldsymbol{d})$, определяемая как конъюнкция априорного $и$ теоретического распределения вероятностей

$$
\sigma(\boldsymbol{m}, \boldsymbol{d})=k \cdot \frac{\rho(\boldsymbol{m}, \boldsymbol{d}) \Theta(\boldsymbol{m}, \boldsymbol{d})}{\mu(\boldsymbol{m}, \boldsymbol{d})},
$$

где $\mu(\boldsymbol{m}, \boldsymbol{d})$ - плотность равномерного распределения вероятностей («функция меры» в колмогоровской тройке определения вероятностного пространства).

Положение (3), известное как формула Тарантолы - Валетта [19], по сути, является обобщением классической теоремы Байеса; отсюда происходит название указанного подхода к решению обратных задач.

Существование и единственность решения обратной задачи в этом подходе гарантируется положением (3); главной проблемой является извлечение из функции $\sigma(\boldsymbol{m}, \boldsymbol{d})$ информации о различных аспектах связи $\boldsymbol{m} \sim \boldsymbol{d}$ в силу (13). Поэтому вместо термина «решение (как процесс) обратной задачи» более целесообразным представляется употребление термина «исследование обратной задачи».

Соотношение (13) носит достаточно общий характер. С его помощью можно, например, определить частные плотности распределения $\sigma_{\boldsymbol{M}}(\boldsymbol{m})$ в пространстве параметров

$$
\sigma_{M}(\boldsymbol{m})=\int_{\boldsymbol{H}_{\boldsymbol{D}}} \sigma(\boldsymbol{m}, \boldsymbol{d}) d \boldsymbol{d}
$$


и $\sigma_{\boldsymbol{D}}(\boldsymbol{d})$ в пространстве данных

$$
\sigma_{D}(\boldsymbol{d})=\int_{\boldsymbol{H}_{\boldsymbol{M}}} \sigma(\boldsymbol{m}, \boldsymbol{d}) d \boldsymbol{m} .
$$

На практике вводят ряд дополнительных гипотез, облегчающих извлечение полезной информации из апостериорной плотности распределения.

1. Представление априорной плотности $\rho(\boldsymbol{m}, \boldsymbol{d})$ в виде

$$
\rho(\boldsymbol{m}, \boldsymbol{d})=\rho_{\boldsymbol{M}}(\boldsymbol{m}) \rho_{\boldsymbol{D}}(\boldsymbol{d})
$$

означает, что информация о распределении исследуемой величины в пространстве параметров получена независимо от соответствующей априорной информации в пространстве данных. Аналогично

$$
\mu(\boldsymbol{m}, \boldsymbol{d})=\mu_{M}(\boldsymbol{m}) \mu_{D}(\boldsymbol{d}),
$$

где $\mu_{\boldsymbol{M}}(\boldsymbol{m}), \mu_{\boldsymbol{D}}(\boldsymbol{d})$ - плотности равномерного распределения вероятностей в пространстве параметров и данных соответственно.

2. Возможность представления теоретической плотности распределения $\Theta(\boldsymbol{m}, \boldsymbol{d})$ по формуле полной вероятности

$$
\Theta(\boldsymbol{m}, \boldsymbol{d})=\theta(\boldsymbol{d} \mid \boldsymbol{m}) \mu_{M}(\boldsymbol{m}) .
$$

В силу (13), (15)-(17), формула (14) преобразуется к виду

$$
\sigma_{M}(\boldsymbol{m})=k \cdot \rho_{\boldsymbol{M}}(\boldsymbol{m}) L(\boldsymbol{m}),
$$

где функция правдоподобия $L(\boldsymbol{m})$ определяется выражением

$$
L(\boldsymbol{m})=\int_{\boldsymbol{H}_{\boldsymbol{D}}} \frac{\rho_{\boldsymbol{D}}(\boldsymbol{d}) \theta(\boldsymbol{d} \mid \boldsymbol{m})}{\mu_{\boldsymbol{D}}(\boldsymbol{d})} d \boldsymbol{d},
$$

Здесь $k$ - нормирующий множитель.

В трёх важных частных случаях удаётся «проинтегрировать» выражение (19) при условии постоянной плотности равномерного распределения $\mu_{\boldsymbol{D}}(\boldsymbol{d})=$ const .

І. В случае, когда «неопределённости моделизации» малы по сравнению с «неопределённостями наблюдения», можно положить

$$
\theta(\boldsymbol{d} \mid \boldsymbol{m})=\delta(\boldsymbol{d}-\boldsymbol{g}(\boldsymbol{m}))
$$

где $\delta(\cdot)$ - дельта-фрункция Дирака, что даёт $L(\boldsymbol{m})=\rho_{\boldsymbol{D}}(\boldsymbol{g}(\boldsymbol{m}))$, и выражение (18) для апостериорной плотности распределения примет вид 


$$
\sigma_{\boldsymbol{M}}(\boldsymbol{m})=k \cdot \rho_{M}(\boldsymbol{m}) \rho_{D}(\boldsymbol{g}(\boldsymbol{m})) .
$$

II. В случае, когда можно пренебречь «неопределённостями наблюдения» по сравнению с «неопределённостями моделизации»,

$$
\rho_{D}(\boldsymbol{d})=\delta\left(\boldsymbol{d}-\boldsymbol{d}_{o b s}\right),
$$

имеем $L(\boldsymbol{m})=\theta\left(\boldsymbol{d}_{o b s} \mid \boldsymbol{m}\right)$, и, соответственно,

$$
\sigma_{\boldsymbol{M}}(\boldsymbol{m})=k \cdot \rho_{\boldsymbol{M}}(\boldsymbol{m}) \theta\left(\boldsymbol{d}_{o b s} \mid \boldsymbol{m}\right) .
$$

III. Гауссовы неопределённости моделизации

$$
\theta(\boldsymbol{d} \mid \boldsymbol{m})=k_{1} \cdot \exp \left\{-\frac{1}{2}(\boldsymbol{d}-\boldsymbol{g}(\boldsymbol{m}))^{T} \boldsymbol{C}_{T}^{-1}(\boldsymbol{d}-\boldsymbol{g}(\boldsymbol{m}))\right\}
$$

и наблюдения

$$
\rho_{\boldsymbol{D}}(\boldsymbol{d})=k_{2} \cdot \exp \left\{-\frac{1}{2}\left(\boldsymbol{d}-\boldsymbol{d}_{o b s}\right)^{T} \boldsymbol{C}_{d}^{-1}\left(\boldsymbol{d}-\boldsymbol{d}_{o b s}\right)\right\} .
$$

В этом случае можно показать, что

$$
\sigma_{\boldsymbol{M}}(\boldsymbol{m})=k \cdot \rho_{\boldsymbol{M}}(\boldsymbol{m}) \cdot \exp \left\{-\frac{1}{2}\left(\boldsymbol{d}_{o b s}-\boldsymbol{g}(\boldsymbol{m})\right)^{T} \boldsymbol{C}_{D}^{-1}\left(\boldsymbol{d}_{o b s}-\boldsymbol{g}(\boldsymbol{m})\right)\right\},
$$

где результирующая ковариационная матрица определяется соотношением

$$
\boldsymbol{C}_{D}=\boldsymbol{C}_{T}+\boldsymbol{C}_{d} .
$$

В случае, когда и для априорной плотности вероятности используется гауссово распределение

$$
\rho_{\boldsymbol{M}}(\boldsymbol{m})=k_{3} \cdot \exp \left\{-\frac{1}{2}\left(\boldsymbol{m}-\boldsymbol{m}_{\text {prior }}\right)^{T} \boldsymbol{C}_{M}^{-1}\left(\boldsymbol{m}-\boldsymbol{m}_{\text {prior }}\right)\right\},
$$

апостериорная плотность распределения равна

$$
\sigma_{M}(\boldsymbol{m})=k \cdot \exp \{-S(\boldsymbol{m})\},
$$

где фрункция невязки $S(\boldsymbol{m})$ определяется соотношением

$$
\mathrm{S}(\mathrm{m})=\frac{1}{2}\left\{\begin{array}{l}
\left(\mathrm{d}_{\mathrm{obs}}-\mathrm{g}(\mathrm{m})\right)^{\mathrm{T}} \mathrm{C}_{\mathrm{D}}^{-1}\left(\mathrm{~d}_{\mathrm{obs}}-\mathrm{g}(\mathrm{m})\right)+ \\
+\left(\mathrm{m}-\mathrm{m}_{\text {prior }}\right)^{\mathrm{T}} \mathrm{C}_{\mathrm{M}}^{-1}\left(\mathrm{~m}-\mathrm{m}_{\text {prior }}\right)
\end{array}\right\} .
$$

В случае линейного отображения $\boldsymbol{g}(\boldsymbol{m})=\boldsymbol{G m}(\boldsymbol{G}$ - матрица преобразования) решение обратной задачи выписывается в явном виде: апо- 
стериорная плотность распределения $\sigma_{\boldsymbol{M}}(\boldsymbol{m})$ является гауссовой с ковариационной матрицей

$$
\widetilde{\boldsymbol{C}}_{\boldsymbol{M}}=\left(\boldsymbol{G}^{T} \boldsymbol{C}_{D}^{-1} \boldsymbol{G}+\boldsymbol{C}_{\boldsymbol{M}}^{-1}\right)^{-1}
$$

и средним значением

$$
\widetilde{\boldsymbol{m}}=\boldsymbol{m}_{\text {prior }}+\widetilde{\boldsymbol{C}}_{\boldsymbol{M}} \boldsymbol{G}^{T} \boldsymbol{C}_{D}^{-1}\left(\boldsymbol{d}_{\text {obs }}-\boldsymbol{G} \boldsymbol{m}_{\text {prior }}\right) .
$$

В случае, когда оператор $\boldsymbol{g}(\cdot)$ «не слишком отличается» от линейного, фоормулы (27), (28) сохраняют своё значение, если в окрестности точки $\boldsymbol{m}=\boldsymbol{m}_{\text {prior }}$ используется линеаризация

$$
\boldsymbol{G}=\left(G_{i j}\right)=\left.\left(\frac{\partial g_{i}}{\partial m_{j}}\right)\right|_{\boldsymbol{m}=\boldsymbol{m}_{\text {prior }}} .
$$

В других случаях для извлечения необходимой информации из апостериорной плотности $\sigma_{\boldsymbol{M}}(\boldsymbol{m})$ необходимо использовать существующий арсенал методов анализа функций многих переменных. На практике в большинстве случаев значения $\sigma_{M}(\boldsymbol{m})$ «значимо» отличаются от нуля лишь в небольших областях в окрестности точек максимума апостериорной гипотезы

$$
L_{p}(\boldsymbol{m})=\frac{\sigma_{M}(\boldsymbol{m})}{\mu_{M}(\boldsymbol{m})},
$$

или, в целях удобства, её логарифма (и, соответственно, точек минимума фрункции невязки $S(\boldsymbol{m}))$

$$
\Psi_{p}(\boldsymbol{m})=\log \left\{\frac{\sigma_{M}(\boldsymbol{m})}{\mu_{M}(\boldsymbol{m})}\right\}=-S(\boldsymbol{m}) .
$$

Для оценки поведения функции $L_{p}(\boldsymbol{m})$ вблизи локальных максимумов может быть использована локальная матрица ковариаций $\widetilde{\boldsymbol{C}}_{M}$, определённая как обратная к гессиану $\boldsymbol{H}$ фуннции $S(\boldsymbol{m})$ :

$$
\widetilde{\boldsymbol{C}}_{M}=(\boldsymbol{H})^{-1}=\left(\frac{\partial^{2} S(\boldsymbol{m})}{\partial \boldsymbol{m}^{2}}\right)^{-1},
$$

или приближение к ней по формулам (29), (27). 
В общем случае для исследования апостериорной плотности распределения применяют методы статистических испытаний (МонтеКарло).

Получение качественных решений $\sigma_{\boldsymbol{M}}(\boldsymbol{m})$ в значительной степени зависит от выбора гибких схем аппроксимации плотностей распределений $\rho_{\boldsymbol{M}}(\boldsymbol{m}), \rho_{\boldsymbol{D}}(\boldsymbol{d}), \theta(\boldsymbol{d} \mid \boldsymbol{m})$, входящих в состав соотношений (21), (22), (24). Подобные схемы должны решать следующие основные задачи.

1. Проверить обоснованность использования соответствующих параметров указанных исходных плотностей распределений. Например, выяснить, не являются ли слишком оптимистическими (переоценёнными) значения среднеквадратических отклонений параметров неизвестного вектора $\boldsymbol{m}$ для заданного вектора наблюдаемых значений $\boldsymbol{d}_{o b s} \boldsymbol{n}$ соответствующей матрицы ковариаций $\boldsymbol{C}_{D}$.

2. Выявить и устранить так называемые «аномальные значения» вектора данных $\boldsymbol{d}_{o b s}$ («выбросы»), использование которых в модели (13) может привести к некачественным решениям.

3. Найти схему дискретизации параметров в пространстве $\boldsymbol{H}_{\boldsymbol{M}}$, которая обеспечивает наилучшую (по крайней мере, «хорошую») разрешающую способность в соответствии с имеющейся информацией о результатах измерений $\boldsymbol{d}_{o b s}$.

В настоящей работе предложена блочная схема аппроксимации плотностей распределений, входящих в обобщённую формулу Байеса, способствующая решению указанных задач.

Блочная модель задачи с распределёнными параметрами. Разобьём векторы параметров $\boldsymbol{m}$ и модельных данных $\boldsymbol{d}$ на блоки (рис. 3):

$$
\begin{gathered}
\boldsymbol{m}=\boldsymbol{m}^{(1)} \otimes \boldsymbol{m}^{(2)} \otimes \ldots \otimes \boldsymbol{m}^{(m)}, \\
\boldsymbol{d}=\boldsymbol{d}^{(1)} \otimes \boldsymbol{d}^{(2)} \otimes \ldots \otimes \boldsymbol{d}^{(d)} .
\end{gathered}
$$

Сделаем следующие предположения относительно введённых блоков параметров и модельных данных.

I. Каждый блок параметров $\boldsymbol{m}^{(i)}=\left\{m_{1}^{(i)}, m_{2}^{(i)}, \ldots, m_{N_{m}^{(i)}}^{(i)}\right\}^{T}$, $i=1,2, \ldots, m$, имеет независимое от других блоков априорное распределение вероятностей с плотностью $\rho_{M}^{(i)}\left(\boldsymbol{m}^{(i)}\right)$.

II. Плотности вероятности $\rho_{D}^{(i)}\left(\boldsymbol{d}^{(i)}\right), i=1,2, \ldots, d$, соответствующие наблюдаемым значениям блоков $\boldsymbol{d}^{(i)}=\left\{d_{1}^{(i)}, d_{2}^{(i)}, \ldots, d_{N_{d}^{(i)}}^{(i)}\right\}^{T}$, также 
независимы между собой (наблюдения данных одного блока проводятся независимо от других блоков).

III. Для каждого из блоков параметров (данных) плотности распределения $\rho_{M}^{(i)}\left(\boldsymbol{m}^{(i)}\right), \rho_{D}^{(i)}\left(\boldsymbol{d}^{(i)}\right)$, могут быть одного из трёх типов (плотности приведены с точностью до постоянного нормирующего множителя).

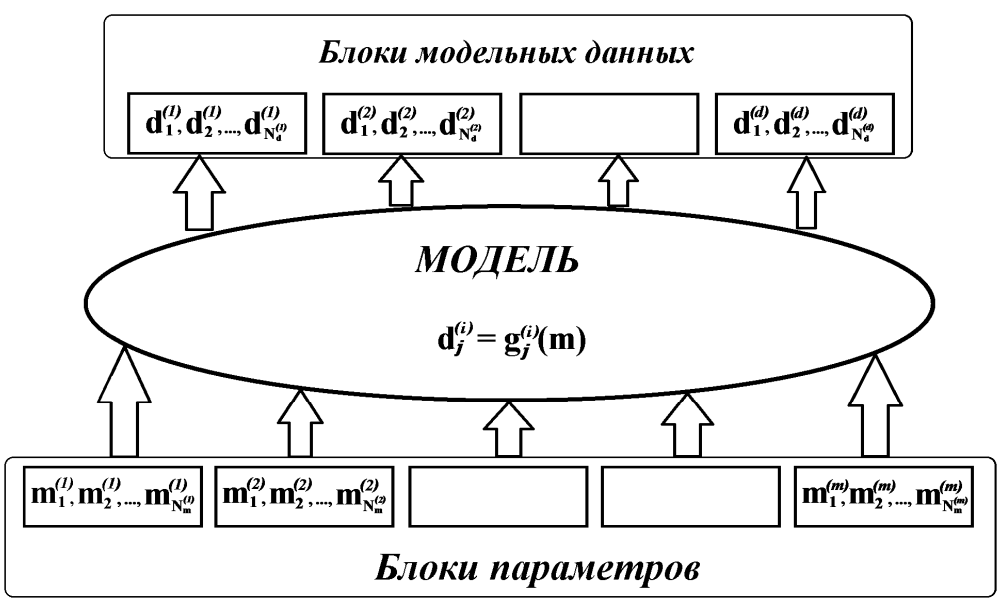

Рис. 3 - Блочная модель обратной задачи

1. Гауссово (Gaussian) распределение.

$$
\begin{aligned}
& \rho_{M}^{(i)}\left(\boldsymbol{m}^{(i)}\right)=\exp \left\{-S_{\boldsymbol{m} \boldsymbol{G}}^{(i)}\left(\boldsymbol{m}^{(i)}\right)\right\}, \\
& \rho_{D}^{(i)}\left(\boldsymbol{d}^{(i)}\right)=\exp \left\{-S_{\boldsymbol{d} \boldsymbol{G}}^{(i)}\left(\boldsymbol{d}^{(i)}\right)\right\} .
\end{aligned}
$$

Здесь

$$
\begin{aligned}
& S_{\boldsymbol{m} \boldsymbol{G}}^{(i)}\left(\boldsymbol{m}^{(i)}\right)=\frac{1}{2} \cdot\left(\boldsymbol{m}^{(i)}-\boldsymbol{m}_{\text {prior }}^{(i)}\right)^{T}\left(\boldsymbol{C}_{M}^{(i)}\right)^{-1}\left(\boldsymbol{m}^{(i)}-\boldsymbol{m}_{\text {prior }}^{(i)}\right), \\
& S_{\boldsymbol{d} \boldsymbol{G}}^{(i)}\left(\boldsymbol{d}^{(i)}\right)=\frac{1}{2} \cdot\left(\boldsymbol{d}^{(i)}-\boldsymbol{d}_{o b s}^{(i)}\right)^{T}\left(\boldsymbol{C}_{D}^{(i)}\right)^{-1}\left(\boldsymbol{d}^{(i)}-\boldsymbol{d}_{o b s}^{(i)}\right)
\end{aligned}
$$

- функции невязки блока параметров и данных соответственно.

В последнем выражении матрица ковариаций для блока данных учитывает неопределённости моделизации и измерений (см. фрормулу (25)).

2. Распределение Лапласа, «длиннохвостое» (Longtail).

$$
\rho_{M}^{(i)}\left(\boldsymbol{m}^{(i)}\right)=\exp \left\{-S_{\boldsymbol{m} \boldsymbol{L}}^{(i)}\left(\boldsymbol{m}^{(i)}\right)\right\},
$$




$$
\rho_{D}^{(i)}\left(\boldsymbol{d}^{(i)}\right)=\exp \left\{-S_{\boldsymbol{d} \boldsymbol{L}}^{(i)}\left(\boldsymbol{d}^{(i)}\right)\right\},
$$

где фрункции невязки блока параметров и данных определяются соотношениями

$$
\begin{aligned}
& S_{\boldsymbol{m} \boldsymbol{L}}^{(i)}\left(\boldsymbol{m}^{(i)}\right)=\sum_{j=1}^{N_{m}^{(i)}} S_{j \boldsymbol{m}}^{(i)}\left(\boldsymbol{m}^{(i)}\right), \\
& S_{\boldsymbol{d} \boldsymbol{L}}^{(i)}\left(\boldsymbol{m}^{(i)}\right)=\sum_{j=1}^{N_{d}^{(i)}} S_{j \boldsymbol{d}}^{(i)}\left(\boldsymbol{d}^{(i)}\right) ;
\end{aligned}
$$

функции невязки $S_{j \boldsymbol{m}}^{(i)}\left(\boldsymbol{m}^{(i)}\right)$ и $S_{j \boldsymbol{d}}^{(i)}\left(\boldsymbol{d}^{(i)}\right)$ для $j$-й переменной выражаются так

$$
\begin{aligned}
S_{j \boldsymbol{m}}^{(i)}\left(\boldsymbol{m}^{(i)}\right) & =\left|\frac{m_{j}^{(i)}-m_{j \text { prior }}^{(i)}}{\sigma_{M j}^{(i)}}\right|, \\
S_{j \boldsymbol{d}}^{(i)}\left(\boldsymbol{d}^{(i)}\right) & =\left|\frac{d_{j}^{(i)}-d_{j o b s}^{(i)}}{\sigma_{D j}^{(i)}}\right| .
\end{aligned}
$$

3. Равномерное распределение (Boxcar).

$$
\begin{gathered}
\rho_{M}^{(i)}\left(\mathbf{m}^{(i)}\right)= \begin{cases}1, \text { если для всех } j=1,2, \ldots N_{m}^{(i)} & S_{j \boldsymbol{m}}^{(i)}\left(\mathbf{m}^{(i)}\right) \leq 1, \\
0, \text { еслидля хотя быодного j } & S_{j \boldsymbol{m}}^{(i)}\left(\mathbf{m}^{(i)}\right)>1 .\end{cases} \\
\rho_{D}^{(i)}\left(\mathbf{d}^{(i)}\right)= \begin{cases}1, \text { если для всех } j=1,2, \ldots N_{d}^{(i)} & S_{j \boldsymbol{d}}^{(i)}\left(\mathbf{d}^{(i)}\right) \leq 1, \\
0, \text { еслидля хотябыодного j } & S_{j \boldsymbol{d}}^{(i)}\left(\mathbf{d}^{(i)}\right)>1 .\end{cases}
\end{gathered}
$$

Вектор параметров $\boldsymbol{m} \in \boldsymbol{H}_{\boldsymbol{M}}$ назовём допустимым относительно Boxcar-блока с индексом $i=1,2, \ldots, m$, если $\rho_{M}^{(i)}\left(\boldsymbol{m}^{(i)}\right)=1$, и не допустимым, если $\rho_{M}^{(i)}\left(\boldsymbol{m}^{(i)}\right)=0$. Аналогично, вектор модельных данных $\boldsymbol{d} \in \boldsymbol{H}_{\boldsymbol{D}}$ является допустимым относительно Boxcar-блока с индексом $i=1,2, \ldots, d$, если $\rho_{D}^{(i)}\left(\boldsymbol{d}^{(i)}\right)=1$, и не допустимым, если $\rho_{D}^{(i)}\left(\boldsymbol{d}^{(i)}\right)=0$. Если же блок не имеет типа Boxcar, то любой вектор будем считать относительно него допустимым. 
IV. Неопределённости моделизации $\theta^{(i)}\left(\boldsymbol{d}^{(i)} \mid \boldsymbol{m}\right)$ для $i$-го блока данных, $i=1,2, \ldots, d$, имеют вид (20):

$$
\theta^{(i)}\left(\boldsymbol{d}^{(i)} \mid \boldsymbol{m}\right)=\delta^{(i)}\left(\boldsymbol{d}^{(i)}-\boldsymbol{g}^{(i)}(\boldsymbol{m})\right) .
$$

В случае блока данных типа Gaussian возможно также использование гауссова закона распределения типа (23); при этом будем считать, что в результирующем распределении (24) в ковариационной матрице $C_{D}^{(i)}$ уже учтены неопределённости моделизации и измерений по формуле (25).

Из (37) и (19) вытекает следующее выражение для фрункции правдоподобия $i$-го блока данных:

$$
L^{(i)}(\boldsymbol{m})=\rho_{D}^{(i)}\left(\boldsymbol{g}^{(i)}(\boldsymbol{m})\right),
$$

$\boldsymbol{g}^{(i)}(\boldsymbol{m})=\left\{g_{1}^{(i)}(\boldsymbol{m}), g_{2}^{(i)}(\boldsymbol{m}), \ldots, g_{N_{d}^{(i)}}^{(i)}(\boldsymbol{m})\right\}^{T}, i=1,2, \ldots, d$. Вектор параметров $\boldsymbol{m}$ будем считать допустимым относительно $i$-го блока модельных данных, если $L^{(i)}(\boldsymbol{m})>0$, и не допустимым, если $L^{(i)}(\boldsymbol{m})=0$.

$\mathrm{V}$. Введённые плотности распределения для блоков параметров определяют «естественную» метрику для каждого блока. А именно, соответствующие нормы для введённых блоков имеют следующий вид.

1. Gaussian. Обобщённая квадратичная норма $\|\cdot\|_{2}$, ассоциированная с ковариационной матрицей $\boldsymbol{C}_{M}^{(i)}$ :

$$
\left\|\boldsymbol{m}^{(i)}\right\|_{2}=\left\{\boldsymbol{m}^{(i) T}\left(\boldsymbol{C}_{M}^{(i)}\right)^{-1} \boldsymbol{m}^{(i)}\right\}^{\frac{1}{2}} .
$$

2. Longtail. Обобщённая суммарная норма $\|\cdot\|_{l}$, ассоциированная с вектором среднеквадратических отклонений $\left\{\sigma_{M}^{(i)}\right\}$ :

$$
\left\|\boldsymbol{m}^{(i)}\right\|_{1}=\sum_{j=1}^{N_{m}^{(i)}}\left|\frac{m_{j}^{(i)}}{\sigma_{M j}^{(i)}}\right| .
$$

2. Boxcar. Обобщённая максимальная («минимаксная») норма $\|\cdot\|_{b_{0}}$, ассоциированная с вектором среднеквадратических отклонений $\left\{\sigma_{M}^{(i)}\right\}$ :

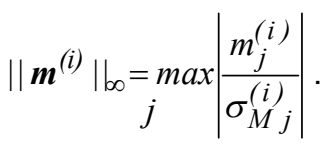


Помимо «естественной», для каждого блока параметров можно задавать иную метрику трёх перечисленных типов.

Получим выражение для апостериорной плотности вероятности (14) распределения вектора модельных параметров $\boldsymbol{m}$ рассматриваемой модели I-V. Множества индексов блоков параметров $I^{m}=\{1,2, \ldots m\}$ и данных $I^{d}=\{1,2, \ldots d\}$ разобьём на подмножества, соответствующие типам блоков Gaussian, Longtail и Boxcar: $I^{m}=I_{G}^{m} \cup I_{L}^{m} \cup I_{B}^{m}$, $I^{d}=I_{G}^{d} \cup I_{L}^{d} \cup I_{B}^{d}$. Обозначим $I_{G L}^{m}=I_{G}^{m} \cup I_{L}^{m}, I_{G L}^{d}=I_{G}^{d} \cup I_{L}^{d}$. Вектор параметров $\boldsymbol{m} \in \boldsymbol{H}_{\boldsymbol{M}}$ будем называть допустимым (относительно модели I-V), если он является допустимым для каждого из блоков параметров и модельных данных, входящих в модель. С учётом (18), а также предположений I- IV, получим

$$
\sigma_{M}(\mathbf{m})=\left\{\begin{array}{l}
k \cdot \exp \{-S(\mathbf{m})\}, \text { если } \mathbf{m}-\text { допустимый вектор }, \\
0, \text { в противном случае. }
\end{array}\right.
$$

Функция невязки $S(\boldsymbol{m})$ определяется соотношением

$$
S(\boldsymbol{m})=S_{\boldsymbol{m}}(\boldsymbol{m})+S_{\boldsymbol{d}}(\boldsymbol{m}),
$$

где фуннции невязки по параметрам $S_{\boldsymbol{m}}(\boldsymbol{m})$ и по данным $S_{\boldsymbol{d}}(\boldsymbol{m})$ определяются как

$$
\begin{gathered}
S_{\boldsymbol{m}}(\boldsymbol{m})=\sum_{i \in I_{G L}^{m}} S_{\boldsymbol{m}}^{(i)}\left(\boldsymbol{m}^{(i)}\right), \\
S_{\boldsymbol{d}}(\boldsymbol{m})=\sum_{i \in I_{G L}^{d}} S_{\boldsymbol{d}}^{(i)}\left(\boldsymbol{g}^{(i)}(\boldsymbol{m})\right) .
\end{gathered}
$$

Невязки для блоков параметров $S_{\boldsymbol{m}}^{(i)}$ и данных $S_{\boldsymbol{d}}^{(i)}$ выражаются в зависимости от типа блока соотношениями (30)-(33), (34), (35).

Во многих случаях для исследования апостериорной плотности (41) необходимо найти точку в пространстве $\boldsymbol{H}_{\boldsymbol{M}}$, соответствующую максимальной апостериорной гипотезе (точку минимума функции $S(\boldsymbol{m})$ ), а также, в случае мультимодальных решений, и аргументы других локальных максимумов функции $\sigma_{M}(\boldsymbol{m})$. Для этой цели обычно используют градиентные методы минимизации невязочной функции (42). Выпишем выражение для градиента этой фрункции, $\hat{\boldsymbol{\gamma}}=\hat{\boldsymbol{\gamma}}^{(1)} \otimes \hat{\boldsymbol{\gamma}}^{(1)} \otimes \ldots \otimes \hat{\boldsymbol{\gamma}}^{(m)}$ для допустимой точки $\boldsymbol{m} \in \boldsymbol{H}_{\boldsymbol{M}}$. Градиент $i$-го блока параметров в силу (42) определится как 


$$
\hat{\gamma}^{(i)}=\hat{\gamma}_{\boldsymbol{m}}^{(i)}+\sum_{k \in I_{G L}^{d}} \hat{\gamma}_{\boldsymbol{d}}^{(k)(i)}, \quad i \in I^{m} .
$$

Блочные градиенты $\hat{\gamma}_{\boldsymbol{m}}^{(i)}, \hat{\gamma}_{\boldsymbol{d}}^{(k)(i)}$ для каждого из типов блоков в силу (32)-(36), (43), (44) определятся соотношениями

$$
\begin{aligned}
& \hat{\boldsymbol{\gamma}}_{\boldsymbol{m}}^{(i)}=\hat{\boldsymbol{\gamma}}_{G \boldsymbol{m}}^{(i)}=\left(\boldsymbol{C}_{M}^{(i)}\right)^{-1}\left(\boldsymbol{m}^{(i)}-\boldsymbol{m}_{\text {prior }}^{(i)}\right), i \in I_{G}^{m}, \\
& \hat{\gamma}_{\boldsymbol{m}}^{(i)}=\hat{\gamma}_{L \boldsymbol{m}}^{(i)}=\left\{\frac{1}{\sigma_{M j}^{(i)}} \operatorname{sgn}\left(\frac{m_{j}^{(i)}-m_{j \text { prior }}^{(i)}}{\sigma_{M j}^{(i)}}\right)\right\}_{j}, j=\overline{1, N_{\boldsymbol{m}}^{(i)}}, i \in I_{L}^{m}, \\
& \hat{\gamma}_{\boldsymbol{m}}^{(i)}=\hat{\gamma}_{B \boldsymbol{m}}^{(i)}=\boldsymbol{0}, i \in I_{B}^{m}, \\
& \hat{\gamma}_{\boldsymbol{d}}^{(k)(i)}=\hat{\boldsymbol{\gamma}}_{G \boldsymbol{d}}^{(k)(i)}=\left(\boldsymbol{G}^{(k)(i)}\right)^{T}\left(\boldsymbol{C}_{D}^{(i)}\right)^{-1}\left(\boldsymbol{g}^{(k)}(\boldsymbol{m})-\boldsymbol{d}_{o b s}^{(k)}\right), k \in I_{G}^{d}, i \in I^{m}, \\
& \hat{\boldsymbol{\gamma}}_{\boldsymbol{d}}^{(k)(i)}=\hat{\boldsymbol{\gamma}}_{L \boldsymbol{d}}^{(k)(i)}=\left(\boldsymbol{G}^{(k)(i)}\right)^{T}\left\{\frac{1}{\sigma_{D j}^{(i)}} \operatorname{sgn}\left(\frac{g_{j}^{(k)}(\boldsymbol{m})-d_{j o b s}^{(k)}}{\sigma_{D j}^{(i)}}\right)\right\}_{j}, k \in \mathrm{I}_{\mathrm{L}}^{\mathrm{d}}, \\
& i \in I^{m}, j=\overline{1, N_{\boldsymbol{d}}^{(k)}}, \\
& \hat{\boldsymbol{\gamma}}_{\boldsymbol{d}}^{(k)(i)}=\hat{\boldsymbol{\gamma}}_{B \boldsymbol{d}}^{(k)(i)}=\mathbf{0}, k \in I_{B}^{d}, i \in I^{m} .
\end{aligned}
$$

Матрицы-блоки производных $\boldsymbol{G}^{(k)(i)}, k \in I^{d}, i \in I^{m}$, имеют вид

$$
\boldsymbol{G}^{(k)(i)}=\left(\boldsymbol{G}^{(k)(i)}\right)_{l j}=\left(\frac{\partial g_{l}^{(k)}(\boldsymbol{m})}{\partial m_{j}^{(i)}}\right), l=\overline{1, N_{\boldsymbol{d}}^{(k)}}, j=\overline{1, N_{\boldsymbol{m}}^{(i)}} .
$$

Вектор скорейшего подъёма $\boldsymbol{\gamma}=\boldsymbol{\gamma}^{(1)} \otimes \boldsymbol{\gamma}^{(2)} \otimes \ldots \otimes \gamma^{(m)}$ имеет в своём составе векторы-блоки $\gamma^{(i)}, i \in I^{m}$, ассоциированные в «естественной» метрике с нормами (38)-(40), которые определяются выражениями

$$
\boldsymbol{\gamma}^{(i)}=\boldsymbol{\gamma}_{G}^{(i)}=\boldsymbol{C}_{M}^{(i)} \hat{\gamma}^{(i)}, \quad i \in I_{G}^{m},
$$




$$
\begin{aligned}
& \gamma^{(\mathrm{i})}=\gamma_{\mathrm{L}}^{(\mathrm{i})}=\left(\hat{\gamma}_{\mathrm{L} \mathrm{j}}^{(\mathrm{i})}\right)_{\mathrm{j}=1, \mathrm{~N}_{\mathrm{m}}^{(\mathrm{i})}}=\left(\begin{array}{l}
\frac{1}{\mathrm{~K}} \cdot \sigma_{\mathrm{M} \mathrm{j}}^{(\mathrm{i})} \cdot \operatorname{sgn}\left(\hat{\gamma}_{\mathrm{j}}^{(\mathrm{i})}\right), \text { если } \\
\mathrm{j}-\text { я компонента является } \\
\text { главной для вектора } \hat{\gamma}^{(\mathrm{i})} ; \\
0 \text { для других компонент }
\end{array}\right)_{\mathrm{j}}, i \in I_{L}^{m}, \\
& \boldsymbol{\gamma}^{(i)}=\boldsymbol{\gamma}_{B}^{(i)}=\left(\hat{\boldsymbol{\gamma}}_{B j}^{(i)}\right)_{j=1, N_{\boldsymbol{m}}^{(i)}}=\left\{\sigma_{M j}^{(i)} \cdot \operatorname{sgn}\left(\hat{\gamma}_{j}^{(i)}\right)\right\}_{j}, \quad i \in I_{B}^{m} .
\end{aligned}
$$

Главной компонентой вектора $\hat{\gamma}^{(i)}$ будет компонента, для которой величина $\sigma_{M j}^{(i)}\left|\hat{\gamma}_{j}^{(i)}\right|$ достигает своего максимального значения, $j=\overline{1, N_{\boldsymbol{m}}^{(i)}} ; K-$ количество главных компонент.

Имея фрормулы для компонент вектора $\boldsymbol{\gamma}$, можно реализовать метод скорейшего спуска следующим образом

$$
\boldsymbol{m}_{n+1}=\boldsymbol{m}_{n}-\mu_{n} \boldsymbol{F}_{n} \gamma_{n},
$$

где $n$ - номер итерации метода; $\boldsymbol{F}_{n}$ - оператор предобуславливания; множитель $\mu_{n}$ может быть найден, например, методом одномерного поиска минимума функции (42).

В случае, когда вектор параметров $\boldsymbol{m} \in \boldsymbol{H}_{\boldsymbol{M}}$ не является допустимым, в процессе реализации метода скорейшего спуска сначала должна быть достигнута (если это возможно) допустимая область. Для этого используется процесс минимизации следующей функции (т.н. «критерий минимакса»)

$$
R(\boldsymbol{m})=\max \left\{R_{\boldsymbol{m}}(\boldsymbol{m}), R_{\boldsymbol{d}}(\boldsymbol{m})\right\},
$$

где

$$
\begin{gathered}
R_{\boldsymbol{m}}(\boldsymbol{m})=\max _{i \in I_{B}^{m}, j=1, N_{\boldsymbol{m}}^{(i)}}\left(\left|\frac{m_{j}^{(i)}-m_{j \text { prior }}^{(i)}}{\sigma_{M j}^{(i)}}\right|\right), \\
R_{\boldsymbol{d}}(\boldsymbol{m})=\max _{i \in I_{B}^{d}, j=1, N_{\boldsymbol{d}}^{(i)}}\left(\left|\frac{g_{j}^{(k)}(\boldsymbol{m})-d_{j o b s}^{(k)}}{\sigma_{D j}^{(i)}}\right|\right) .
\end{gathered}
$$

Некоторые численные результаты. Рассмотрим упругий двуслойный пакет в фрорме квадрата со стороной $а$ в безразмерных координа- 
тах $\tilde{x}=x / a, \tilde{y}=y / a$ (рис. 2) с условными толщинами слоёв $\tilde{h}_{1}=h_{1} / a=1 / 2 ; \tilde{h}_{2}=h_{2} / a=1 / 2$ (нумерация слоёв начинается с самого нижнего). Материалы слоёв пакета считается изотропно-упругими с модулями сдвига $G_{1}=2 G, G_{2}=G$ и коэффрициентами Пуассона $\mu_{1}=0,25$, $\mu_{2}=0,35$ соответственно. Рассматривается плоская деформация пакета, который находится под действием равномерно распределённой по верхней (прямолинейной) поверхности нагрузки интенсивности $q$. Левая боковая граница пакета гладко опёрта о жёсткую стенку, правая свободная. Слои пакета считаются жёстко сцепленными («склеенными») между собой; нижний слой пакета может скользить без трения по жёсткому основанию.

Неизвестную границу раздела между слоями будем аппроксимировать квадратичным сплайном $\tilde{y}=\tilde{h}_{1}+S_{1}(\tilde{x})$, построенном на узлах интерполяции $\tilde{x}_{i}=i h_{m}, i=-1,0,1,2, \ldots, N_{m}-2, h_{m}=1 /\left(N_{m}-2\right)$. Фиктивный узел $\tilde{x}_{-1}=-h_{m}$ введён с целью обеспечить возможность задания произвольного значения угла наклона кривой $\tilde{y}=\tilde{h}_{1}+S_{1}(\tilde{x})$ в точке $\tilde{x}_{0}=0$; начальное условие для построения сплайна имеет вид $S_{1}^{\prime}\left(\widetilde{x}_{-1}\right)=0$. Вектор параметров $\boldsymbol{m}$ задачи образован одним блоком $\boldsymbol{m}^{(1)}=\left\{m_{1}^{(1)}, m_{2}^{(1)}, \ldots, m_{N_{m}}^{(1)}\right\}^{T}=\boldsymbol{s}^{(1)}=\left\{s_{-1}^{(1)}, s_{0}^{(1)}, \ldots, s_{N_{m}-2}^{(1)}\right\}^{T}$, элементы которого представляют собой значения функции $S_{1}(\widetilde{x})$ в соответствующих узлах интерполяции.

Вектор модельных данных $\boldsymbol{d}$ задачи также состоит из одного блока $\boldsymbol{d}^{(1)}=\left\{d_{1}^{(1)}, d_{2}^{(1)}, \ldots, d_{N_{d}}^{(1)}\right\}^{T}=\left\{\widetilde{v}_{y 0}, \widetilde{v}_{y 1}, \ldots, \widetilde{v}_{y N_{d}-1}\right\}^{T}$, где $\widetilde{v}_{y j}-$ безразмерные вертикальные перемещения точек верхней поверхности пакета в узлах равномерного разбиения $\tilde{x}_{j}=j h_{d}, j=0,1,2, \ldots, N_{d}-1$, $h_{d}=1 /\left(N_{d}-1\right)$ :

$$
\widetilde{v}_{y j}=\widetilde{v}_{y}\left(\tilde{x}_{j}\right)=\frac{v_{y}\left(\tilde{x}_{j}\right) G}{a q} .
$$

Здесь и в дальнейшем под «решением обратной задачи» будем понимать точку в пространстве параметров $\boldsymbol{H}_{\boldsymbol{M}}$, соответствующую максимальной апостериорной гипотезе. Рассмотрим задачу уточнения параметра среднеквадратического отклонения неопределённости наблюдаемых данных. Для этого зададимся определённым значением вектора параметров $\quad \mathrm{m}^{(1)}=\{0,025 ; 0,00625 ;-0,00625 ;-0,025 ; 0 ; 0,025\}^{\mathrm{T}}$ $\left(N_{m}=6\right)$, для которого вычислим соответствующее поле перемещений $\left(\widetilde{v}_{x}, \tilde{v}_{y}\right)$. Вопросы построения сеток для решения указанной «прямой 
задачи», а также настройки параметров многосеточного итерационного алгоритма её решения рассмотрены в работах [12], [11]. Сформируем вектор наблюдаемых данных $\boldsymbol{d}_{o b s}$, взяв значения перемещений $\widetilde{v}_{y}$ в соответствующих узлах равномерного разбиения верхней поверхности пакета $\left(N_{d}=9\right)$ и наложив на них случайный гауссов «шум» с параметром среднеквадратического отклонения $\sigma_{\mathrm{D}}^{\text {noise }}=0,001$ (одинаковым для всех точек) и автокорреляционной функцией Лапласа $\rho(x)=\exp (-x / l)$ $(l=0,7)$. Сформулируем обратную задачу, присвоив блокам параметров и данных неопределённость типа Boxcar. Для неопределённости блока параметров примем $\sigma_{\mathrm{M}}=0,1$ (также одинаковое для всех точек) и нулевое среднее значение для всех параметров, а значение $\sigma_{D}$ для блока данных будем варьировать, каждый раз находя решение с минимальным значением $R$ критерия (45). На рис. 4, а изображена зависимость значения $R$ от величины $\sigma_{D}$, а на рис. 4,б - решение обратной задачи для $\sigma_{\mathrm{D}}=0,0016$ (показано жирной линией) и заданное «точное» решение (тонкая линия).

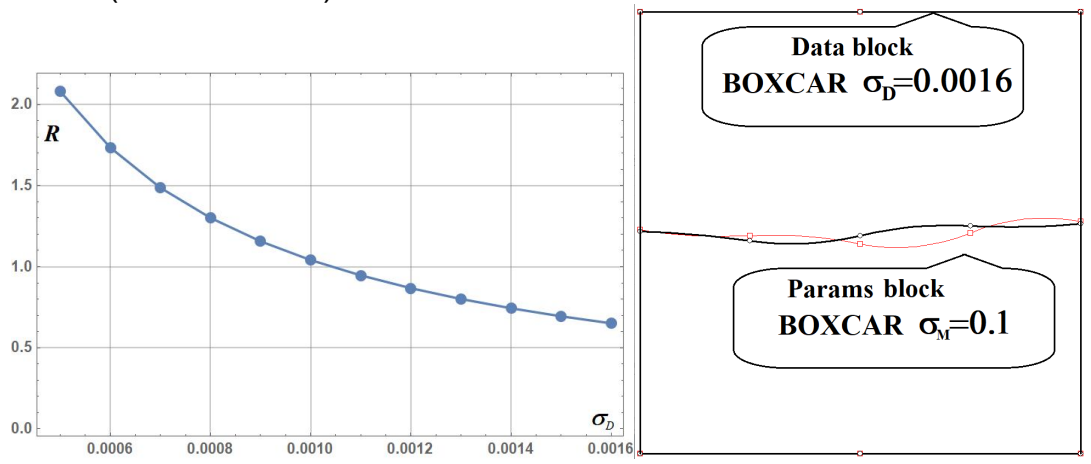

a)

б)

Рис. 4 - Зависимость значения критерия минимакса $R$ (45) от величины $\sigma_{D}(\mathrm{a})$; приближённое решение обратной задачи (жирная линия) и «точное решение» (тонкая линия) (б)

Невысокое качество решения обратной задачи (рис. 4,б) связано с (относительно) высокой величиной «шума» данных. Однако такая модель позволяет достаточно точно оценить «амплитуду» такого шума (абсцисса точки пересечения графика на рис. 4,a с горизонтальной прямой $R=1$ ).

В случае, когда наблюдаемые данные определены достаточно точно, есть возможность оценить также «размах колебаний» значений параметров. Рассмотрим аналогичную модель, когда на данные не нало- 
жен шум, блоки данных и параметров имеют тип Boxcar, $\sigma_{\mathrm{D}}=0,0001$ a значение $\sigma_{M}$ варьируется. Решение обратной задачи снова определяется с использованием критерия минимакса (45). На рис. 5 изображена зависимость $R=R\left(\sigma_{M}\right)$ и приближённое решение обратной задачи при $\sigma_{\mathrm{M}}=0,1$.

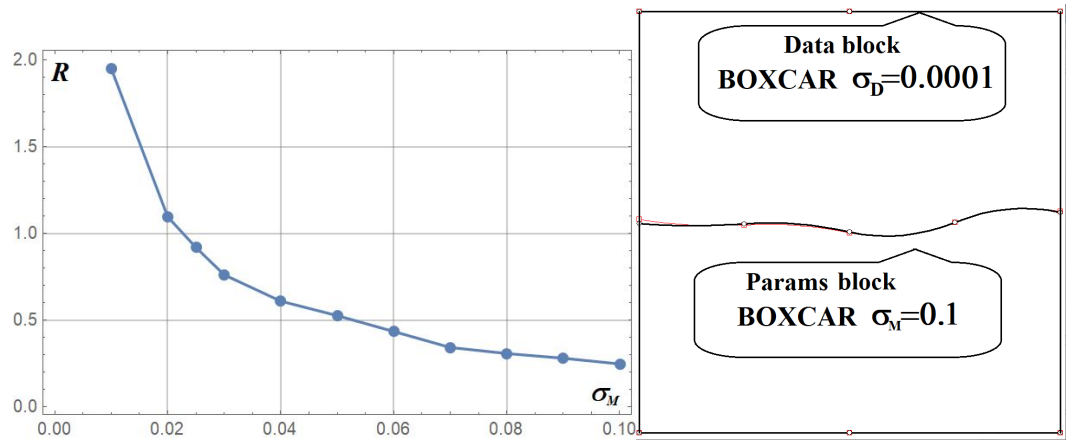

a)

б)

Рис. 5 - График зависимости $R=R\left(\sigma_{M}\right)$ (а); приближённое решение обратной задачи (жирная линия) и «точное решение» (тонкая линия) (б)

Рассмотрим задачу определения аномальных значений («выбросов») наблюдаемых данных. В рассматриваемой задаче внесём возмущение величиной 0,001 в «точное» значение наблюдаемого данного $d_{4}^{(1)}=\tilde{v}_{y 3}$ для $\tilde{x}_{3}=0,375$.

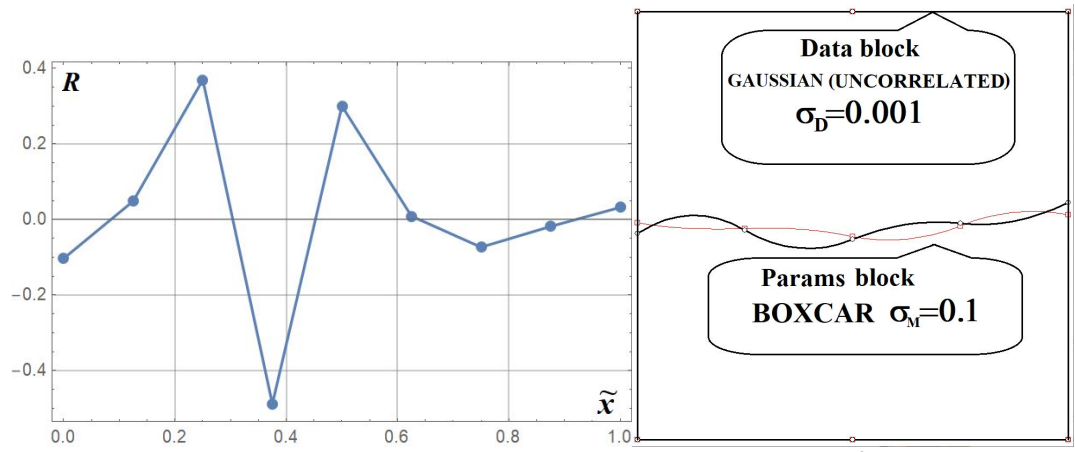

a)

Рис. 6 - Модель "Gaussian”:

б)

график нормализованной невязки по данным $R_{d}^{(1)}\left(\boldsymbol{m}, \tilde{x}_{j}\right)(\mathbf{a})$;

приближённое решение обратной задачи (жирная линия) и «точное решение» (тонкая линия) (б)

Результаты расчётов по двум моделям представлены на рис. 6 и рис. 7. Для первой модели неопределённость данных принимается 
имеющей тип Gaussian c единичной матрицей корреляций (некоррелированный или «белый» шум) и среднеквадратическим отклонением $\sigma_{\mathrm{D}}=0,001$. Во второй модели неопределённость данных рассматривается как Longtail с таким же среднеквадратическим отклонением $\sigma_{\mathrm{D}}=0,001$. Для неопределённости параметров обеих моделей используется тип Boxcar с нулевым средним для всех параметров и среднеквадратическим отклонением $\sigma_{\mathrm{M}}=0,1$ (по сути, случай отсутствия «регуляризации»).

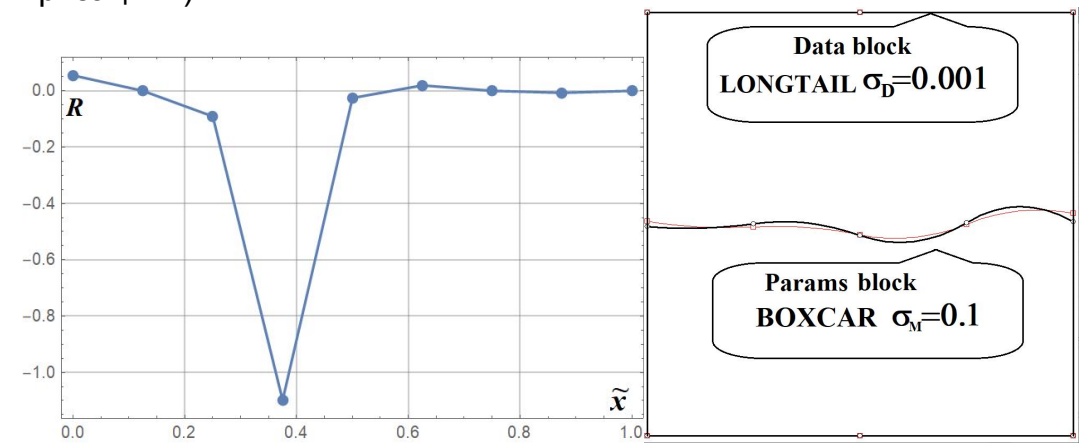

a)

б)

Рис. 7 - Модель "Longtail":

график нормализованной невязки по данным $R_{d}^{(1)}\left(\boldsymbol{m}, \tilde{x}_{j}\right)(\mathbf{a})$;

приближённое решение обратной задачи (жирная линия)

и «точное решение» (тонкая линия) (б)

На рис. 6,а и рис. 7,а представлены графики величины нормализованной невязки

$$
R_{\boldsymbol{d}}^{(1)}\left(\boldsymbol{m}, \tilde{x}_{j}\right)=\frac{g_{j}^{(1)}(\boldsymbol{m})-d_{j o b s}^{(1)}}{\sigma_{D j}^{(1)}}, j=1,2, \ldots, N_{d},
$$

в узлах блока данных для двух рассмотренных моделей. Из графиков видно, что модель "Gaussian" «размазывает» величину возмущения («выброса») по соседним точкам, в то время как модель "Longtail” позволяет точно локализовать «выброс» и оценить его величину. Кроме того, из сравнения рис. 6,б и, рис. 7,б можно видеть, что решение обратной задачи в модели "Longtail", в отличие от модели "Gaussian", coхраняет приемлемое качество.

На рис. 8 проиллюстрировано влияние уровня «шума данных» на качество решения обратной задачи в отсутствие «регуляризации».

Рассматривается модель с неопределённостью данных типа «некоррелированный Gaussian» со среднеквадратическим отклонением $\sigma_{\mathrm{D}}=0,0001$; неопределённость параметров имеет тип Boxcar c пара- 
метром $\sigma_{\mathrm{M}}=0,1$. На точные значения наблюдаемых данных наложен «белый шум» (неопределённость типа «некоррелированный Gaussian») с последовательно увеличивающимся параметром среднеквадратического отклонения $\sigma_{D}^{\text {noise }}$. Результаты решения обратной задачи для некоторых значений этого параметра приведены на рис. 8.

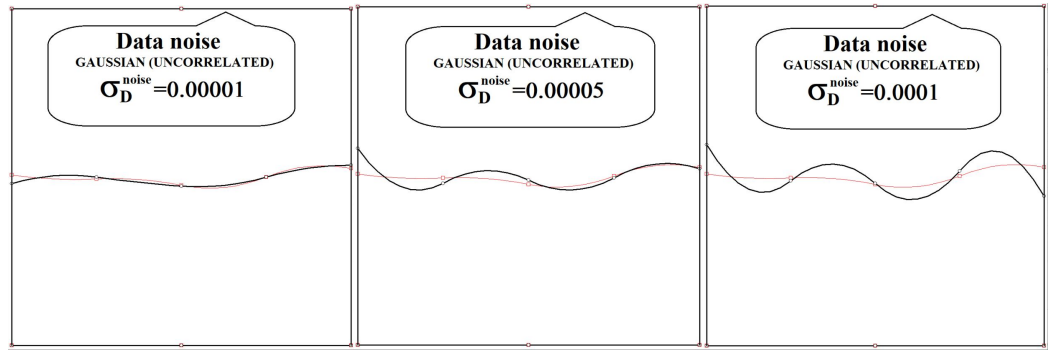

a)

б)

B)

Рис. 8 - Чувствительность решения обратной задачи

к уровню «шума» наблюдаемых данных

Отсутствие «регуляризации» для указанных значений параметра $\sigma_{D}^{n o i s e}$ означает, что здесь не «срабатывают» ограничения на значения параметров, задаваемые неопределённостью Boxcar, т.е. невязка (46) на решении обратной задачи строго меньше единицы. Из рис. 8 вытекает, что регуляризация может потребоваться при значениях параметра шума $\sigma_{\mathrm{D}}^{\text {noise }}>0,00005$.

Рассмотрим вопрос о влиянии уровня регуляризации на качество решения обратной задачи. Возьмём модель с неопределённостями типа «некоррелированный Gaussian» с параметром среднеквадратического отклонения $\sigma_{\mathrm{D}}=0,0005$ для наблюдаемых данных и накладываемого на них случайного «шума». Неопределённость параметров также имеет тип «некоррелированный Gaussian»; параметр $\sigma_{M}$ характеризует степень «регуляризации».

Естественно ожидать, что наилучшие результаты применения регуляризации получатся в случае, когда невязки по параметрам $S_{\boldsymbol{m}}(\boldsymbol{m})$ и данным $S_{\boldsymbol{d}}(\boldsymbol{m})$ будут примерно одинаковы (по порядку величины). На рис. 9 показано распределение в процентном отношении значений функций (43) и (44) на решении обратной задачи в суммарной невязке (42) в зависимости от величины параметра регуляризации б. фика следует, что соответствующие значения параметра находятся в промежутке $\sigma_{\mathrm{M}} \in[0,005 ; 0,02]$. Это именно те величины, для которых «точные» значения параметров обратной задачи могли бы вероятно быть «выборочными» из априорного распределения с соответствую- 
щим параметром. Рис. 10 свидетельствует, насколько важно правильно «угадать» параметры априорного распределения.

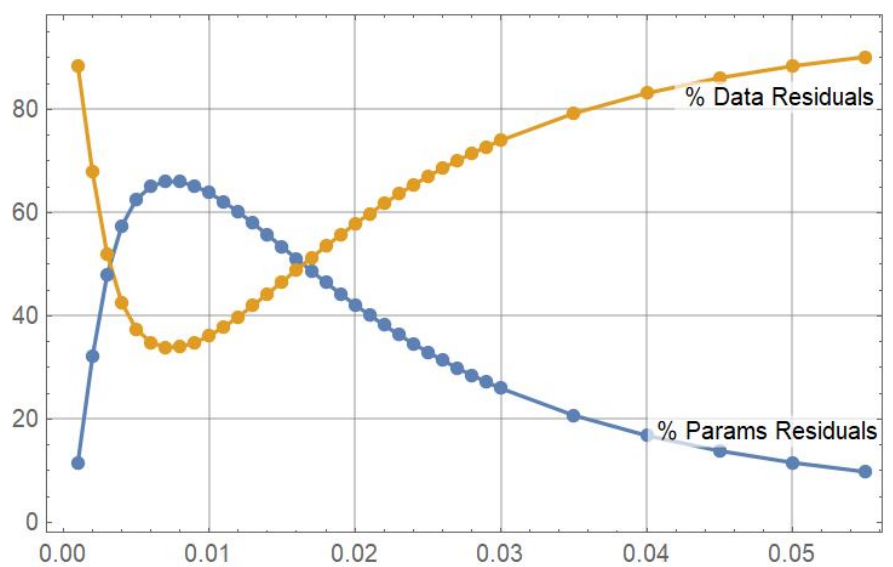

Рис. 9 - Зависимость распределения (в \%) частей $S_{\boldsymbol{m}}(\boldsymbol{m})$ и $S_{\boldsymbol{d}}(\boldsymbol{m})$ в общей сумме значения функции невязки $S(\boldsymbol{m})$ от параметра регуляризации $\sigma_{M}$

На рис. 10,а показан случай чрезмерной регуляризации, когда решение обратной задачи «притянуто» к априорному среднему. Случай 10,в, напротив, свидетельствует о недостаточной регуляризации, которая не препятствует усилению «шума» «обратным оператором» $\boldsymbol{g}^{-1}(\cdot)$. Случай же 10,б показывает подходящий уровень регуляризации, приводящий к наиболее качественному решению обратной задачи.

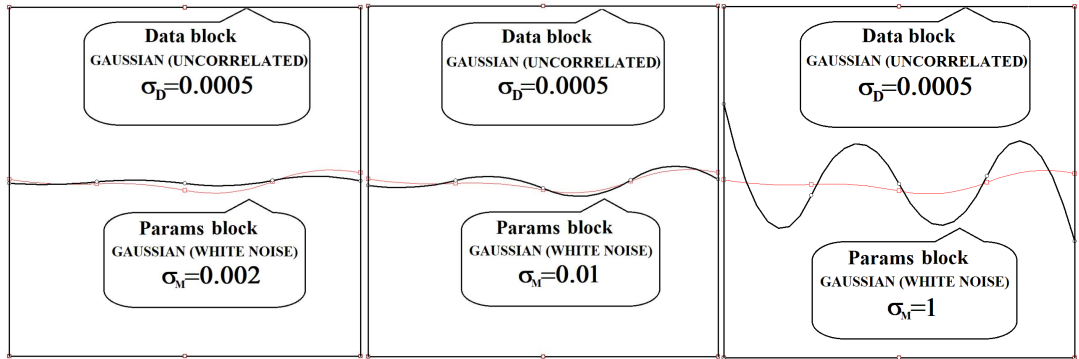

a)

б)

B)

Рис. 10 - Влияние параметра априорного распределения

$\sigma_{M}$ («параметра регуляризации») на качество решения обратной задачи: чрезмерная (а), подходящая (б), недостаточная (в) регуляризация 
Выводы. Предложенный блочно-параметрический подход к решению обратных задач в рамках «информационно-вероятностной» концепции был реализован в задачах определения неизвестных границ упругого слоистого пакета по заданным перемещениям некоторых точек его верхней (нагруженной) поверхности. Полученные результаты свидетельствуют, что предложенный подход является весьма перспективным для исследования решений широкого класса обратных задач, в том числе и задач с неизвестными параметрами геометрии рассматриваемой области.

\section{Перспективы дальнейших исследований.}

1. Выяснить влияние «внутреннего шума» численного метода решения «прямой задачи» на качество решения «обратной задачи» в рамках предложенного класса моделей. Поскольку основная часть вычислительной работы приходится именно на приближённое решение «прямой задачи», разумный выбор уровня её дискретизации позволит достичь приемлемого времени решения «обратной задачи». Такой учёт «внутреннего шума» в рассматриваемом классе моделей может быть осуществлён (в предположении нормальности такого «шума») в соответствии с формулами (23)-(26).

2. Исследовать, как меняется качество решения обратной задачи в зависимости от глубины расположения слоя пакета. Очевидно, оно будет ухудшаться с глубиной. Представляет интерес выяснить, для какого уровня «шума данных» разрешающая способность предложенных моделей будет достаточной для более-менее удовлетворительного определения параметров глубинных слоёв пакета, а также гуртового основания.

3. Определить возможности современного инструментария для приближения функций многих переменных (нейронные сети и т.д.) для оперативной аппроксимации решения обратной задачи. Этот вопрос имеет особую актуальность для высокоскоростных сканирующих устройств, позволяющих сразу отображать исследуемые параметры слоистого основания.

\section{БИБЛИОГРАФИЧЕСКИЕ ССЫЛКИ}

1. Трофимов О.В. Многосеточные итерационные алгоритмы решения граничных задач для упругих и упругопластических слоистых пакетов с криволинейными границами // Вісник Академії митної служби України. Серія: Технічні науки. 2016. №1 (55). С. $119-137$.

2. Трофимов О.В., Петрова Ю.В. Многосеточные итерационные алгоритмы построения сеток для упругих и упругопластических слоистых пакетов // Вісник Академії митної служби України. Серія: Технічні науки. 2015. №2(54). С. 69-81. 2015.

3. Animesh Das. Analysis of pavement structures. CRC Press, Taylor \& Francis Group,

4. Backus G., Gilbert F. Uniqueness in the inversion of inaccurate gross Earth data // Philos. Trans. Royal Soc.: London. 1970. Vol. 266. P. 123-192.

5. Berkovic G., Shafir E. Optical methods for distance and displacement measurements // Advances in Optics and Photonics. 2012. Vol. 4. P. 441-471. 
6. Ceylan H., Gopalakrishnan K. Finite element based hybrid evolutionary optimization approach to solving rigid pavement inversion problem // Engineering with Computers. 2014. Vol. 30. P. 1-13. https://doi.org/10.1007/s00366-012-0281-y.

7. Flintsch G., Katicha S., Bryce J., Ferne B., Nell S. Diefenderfer B. Assessment of Continuous Pavement Deflection Measuring Technologies. // SHRP 2 Report S2-R06FRW-1. Washington, DC: 2013. TRB, National Academy of Sciences.

8. Göktepe Burak, Altun Selim. Artificial Intelligence Applications in the Backcalculation of the Mechanical Properties of Flexible Pavements // https://www.researchgate.net/publication/242632116_Artificial_Intelligence_Applications_in the_Backcalculation_of_the_Mechanical_Properties_of_Flexible_Pavements\#fullTextFile Content. 2009.

9. Hadidi R., Gucunski N. Probabilistic Inversion: A New Approach to Inversion Problems in Pavement and Geomechanical Engineering // In: Gopalakrishnan K., Ceylan H., Attoh-Okine N.O. (eds) Intelligent and Soft Computing in Infrastructure Systems Engineering. Studies in Computational Intelligence. 2009. Vol. 259. P. 21-45. Springer, Berlin, Heidelberg.

10. Ji. Richard, Siddiki Nayyarzia, Nantung Tommy, Kim Daehyeon. Evaluation of Resilient Modulus of Subgrade and Base Materials in Indiana and Its Implementation in MEPDG // The Scientific World Journal. 2014. 372838. 10.1155/2014/372838. P. 1-21.

11. Morosiuk G., Riley M., Odoki J.B. Modelling Road Deterioration and Works Effects. Version 2, HDM-4. // The Highway Development and Management Series. 2004. Vol. 6. Paris: World Road Association.

12. Nikolaides A. Highway Engineering. Pavements, Materials and Control of Quality. CRC Press, Taylor \& Francis Group. 2014.

13. Park S., Park H.M., Hwang J. Application of Genetic Algorithm and Finite Element Method for backcalculating layer moduli of flexible pavements // KSCE J. Civ. Eng. 2010. Vol. 14. P. 183-190. https://doi.org/10.1007/s12205-010-0183-8.

14. Per Ullidtz Pavement Analysis. Amsterdam: Elsevier. 1987.

15. Saltan M., Terzi S. Backcalculation of Pavement Layer Thickness and Moduli Using Adaptive Neuro-fuzzy Inference System // In: Gopalakrishnan K., Ceylan H., AttohOkine N.O. (eds) Intelligent and Soft Computing in Infrastructure Systems Engineering. Studies in Computational Intelligence. 2009. Vol. 259. Springer, Berlin, Heidelberg. P. 177204.

16. Saltan M., Terzi S., Küçüksille E.U. Backcalculation of pavement layer moduli and Poisson's ratio using data mining // Expert Systems With Applications. 2011. Vol. 38, 3. P. 2600-2608.

17. Saric A., Pozder M. Artificial Neural Networks Application in the Backcalculation Process of Flexible Pavement Layers Elasticity Modulus. // In: Hadžikadić M., Avdaković S. (eds) Advanced Technologies, Systems, and Applications II. IAT 2017. Lecture Notes in Networks and Systems. Vol. 28. Springer, Cham. P. 549-559.

18. Tarantola A. Inverse Problem Theory and Methods for Model Parameter Estimation. Philadelphia: SIAM, 2005. Society for Industrial and Applied Mathematics.

19. Tarantola A., Valette B., Generalized nonlinear inverse problems solved using the least-squares criterion // Rev. Geophys. Space Phys., 1982. Vol. 20, No 2. P. 219-232.

20. Tikhonov A.N., Arsenin V.Y. Solutions of III-Posed Problems // John Wiley and Sons. 1977.

21. Zhang Y., Roesler J.R., Huang Z. A method for evaluating CRCP performance based on edge-loaded FWD test. Mater Struct 53, 46 (2020). https://doi.org/10.1617/s11527-020-01481-0. Art. no. 46. 
О. В. Трофрімов, канд. фріз.-мат. наук

\title{
АНАЛІЗ РОЗВ'ЯЗКІВ ОБЕРНЕНИХ ЗАВДАЧ ОЦІНКИ СТРУКТУРНИХ І ФУНКЦІОНАЛЬНИХ ХАРАКТЕРИСТИК ДОРОЖНІХ ПОКРИТТІВ
}

\begin{abstract}
Запропоновано метод оцінки механічних i геометричних параметрів шаруватих основ, що ґрунтується на розв'язанні обернених задач для багатошарових пружних пакетів на базі інформаційно-імовірнісного (байєсівського) підходу. Суть методу полягає в блочно-параметричному наближенні апріорної щільності ймовірності та функції правдоподібності в просторі параметрів і модельних даних задачі.

Ключові слова: дорожні покриття; оцінка структурних параметрів; обернені задачі; імовірнісний підхід.
\end{abstract}

Своєчасна діагностика параметрів багатошарових основ (фундаментів будівель і споруд, дорожніх покриттів, злітно-посадкових смуг і т. п.) грає важливу роль в забезпеченні безпечного руху транспортних засобів. Запропоновано метод оцінки механічних і геометричних параметрів таких основ на базі розв'язання обернених задач для багатошарових пружних і пружно-пластичних пакетів. В якості вихідних даних для таких задач використовуються виміряні зміщення (або швидкості) певних точок на поверхні основи. Метод заснований на інформаційноімовірнісному підході до аналізу обернених задач, завданням якого $є$ отримання апостеріорної щільності ймовірності в просторі невідомих параметрів. Суть методу полягає в блочно-параметричному наближенні апріорної щільності ймовірності та функції правдоподібності в просторі параметрів і модельних даних задачі. Метод дозволяє оцінити параметри апріорного розподілу діагностованих величин, ідентифрікувати і виключити «викиди» виміряних даних зі створеної моделі, а також побудувати апостеріорну оцінку щільності ймовірності невідомих параметрів 3 прийнятною роздрібною здатністю. Як приклад застосування запропонованого методу розглянуто задачу визначення геометричних характеристик пружного двошарового пакета (визначення фрорми границь розділу шарів). Границі розділу шарів апроксимуються квадратичним сплайном; параметри такого сплайну розглядалися в якості вектора невідомих параметрів оберненої задачі. Розглянуто питання визначення параметрів випадкового "шуму», накладеного на дані спостережень, а також оцінки параметрів можливої варіації невідомих параметрів. Також розглянуті питання локалізації та оцінки амплітуди одиничного «викиду» в блоці даних задачі. Проаналізовано вплив величини «шуму», накладеного на дані, на якість отриманого розв'язку оберненої задачі; розглянуто питання знаходження параметрів оптимальної «регуляризації», тобто величин, що характеризують апріорний розподіл невідомих параметрів задачі.

Запропонований підхід до розв'язання обернених задач для шаруватих пакетів може бути використаний для створення обладнання нового покоління, призначеного для неруйнівного контролю і оцінки стану до- 
рожнього покриття, злітно-посадкової смуги і фундаментів штучних споруд. Може бути розроблено відповідне програмне забезпечення для таких високошвидкісних скануючих пристроїв, які дозволяють выдразу відображати діагностовані параметри шаруватої основи.

UDC 519.6, 625.7I.8 (075.8)

\author{
A. V. Trofimov, PhD (Phys.-Math.) \\ INVERSE PROBLEM ANALYSIS FOR \\ NON-DESTRUCTIVE EVALUATION OF STRUCTURAL \\ PAVEMENT CHARACTERISTICS
}

\begin{abstract}
The method of estimating the mechanical and geometric parameters of layered foundations based on the inverse problems solutions for multi-layered elastic packets is proposed. The core of the method is the block-parametric-type approximation of the posterior probability in the parameters and model data space of the problem.
\end{abstract}

Keywords: pavement; non-destructive evaluation; inverse problems; probabilistic approach.

Timely diagnostics of the parameters of multilayer foundations (foundations of buildings and structures, pavements, runways, etc.) plays an important role in ensuring the safe movement of vehicles. A method for assessing the mechanical and geometric parameters of such foundations on the basis of solving inverse problems for multilayer elastic and elastoplastic packets is proposed. As the initial data for such problems, the measured displacements (or velocities) of certain points on the base foundation are used. The method is based on an informational-probabilistic approach to the analysis of inverse problems, the task of which is to obtain a posterior probability density in the space of unknown parameters. The essence of the method lies in the blockparametric approximation of the a priori probability density and likelihood function in the space of parameters and model data of the problem. The method allows estimating the parameters of the a priori distribution of diagnosed values, identifying and eliminating outliers of measured data from the created model, and also constructing an a posteriori estimate of the probability density of unknown parameters with an acceptable resolution. As an example of the application of the proposed method, the problem of determining the geometric characteristics of an elastic two-layer packet (determining the shape of the interface between the layers) is considered. The layer interface was approximated by a quadratic spline; the parameters of such a spline were considered as a vector of unknown parameters of the inverse problem. The problems of determining the parameters of random "noise" superimposed on the observational data, as well as estimating the parameters of a possible variation of unknown parameters, are considered. The issues of localization and estimation of the amplitude of a single "outlier" in the problem's data block are also considered. In conclusion, the influence of the "noise" value superimposed on the data on the quality of the obtained solu- 
tion of the inverse problem is analyzed; the question of finding the parameters of optimal "regularization" (i.e., appropriate values characterizing the a priori distribution of unknown parameters of the problem) is considered.

The proposed approach to solving inverse problems for layered packages can be used to create a new generation of equipment designed for non-destructive testing and assessment of the condition of the road surface, runway and foundations of artificial structures. Corresponding software can be developed for such high-speed scanning devices that allow on-the-fly display of the diagnosed parameters of the layered foundation.

\section{REFERENCES}

1. Trofimov A.V. Multigrid iterative algorithms for layered elastic and elasto-plastic foundations with curvilinear boundaries' boundary-value problems. Dnipro: Systems and Technologies, № 1 (55), 119-137. (2016)

2. Trofimov A.V., Petrova Y.V. Multigrid iterative grid generation algorithms for layered elastic and elasto-plastic foundations' problems. Dnipro: Systems and Technologies, № 2 (54), 69-80 (2015)

3. Animesh Das. Analysis of pavement structures. CRC Press, Taylor \& Francis Group, 2015.

4. Backus G., Gilbert F. Uniqueness in the inversion of inaccurate gross Earth data. // Philos. Trans. Royal Soc.: London, 1970. vol. 266, 123-192.

5. Berkovic G., Shafir E. Optical methods for distance and displacement measurements. // Advances in Optics and Photonics. 2012, vol. 4. P. 441-471.

6. Ceylan H., Gopalakrishnan K. Finite element based hybrid evolutionary optimization approach to solving rigid pavement inversion problem. // Engineering with Computers. 2014. vol. 30, P. 1-13. https://doi.org/10.1007/s00366-012-0281-y.

Flintsch G., Katicha S., Bryce J., Ferne B., Nell S. Diefenderfer B. Assessment of Continuous Pavement Deflection Measuring Technologies. // SHRP 2 Report S2-R06FRW-1. Washington, DC: 2013. TRB, National Academy of Sciences.

8. Göktepe Burak, Altun Selim. Artificial Intelligence Applications in the Backcalculation of the Mechanical Properties of Flexible Pavements // https://www.researchgate.net/publication/242632116_Artificial_Intelligence_Applications_in the_Backcalculation_of_the_Mechanical_Properties_of_Flexible_Pavements\#fullTextFile Content. 2009.

9. Hadidi R., Gucunski N. Probabilistic Inversion: A New Approach to Inversion Problems in Pavement and Geomechanical Engineering // In: Gopalakrishnan K., Ceylan H., Attoh-Okine N.O. (eds) Intelligent and Soft Computing in Infrastructure Systems Engineering. Studies in Computational Intelligence. 2009. Vol. 259. P. 21-45. Springer, Berlin, Heidelberg.

10. Ji. Richard, Siddiki Nayyarzia, Nantung Tommy, Kim Daehyeon. Evaluation of Resilient Modulus of Subgrade and Base Materials in Indiana and Its Implementation in MEPDG // The Scientific World Journal. 2014. 372838. 10.1155/2014/372838. P. 1-21.

11. Morosiuk G., Riley M., Odoki J.B. Modelling Road Deterioration and Works Effects. Version 2, HDM-4. // The Highway Development and Management Series. 2004. Vol. 6. Paris: World Road Association.

12. Nikolaides A. Highway Engineering. Pavements, Materials and Control of Quality. CRC Press, Taylor \& Francis Group. 2014.

13. Park S., Park H.M., Hwang J. Application of Genetic Algorithm and Finite Element Method for backcalculating layer moduli of flexible pavements // KSCE J. Civ. Eng. 2010. Vol. 14. P. 183-190. https://doi.org/10.1007/s12205-010-0183-8.

14. Per Ullidtz Pavement Analysis. Amsterdam: Elsevier. 1987. 
15. Saltan M., Terzi S. Backcalculation of Pavement Layer Thickness and Moduli Using Adaptive Neuro-fuzzy Inference System // In: Gopalakrishnan K., Ceylan H., AttohOkine N.O. (eds) Intelligent and Soft Computing in Infrastructure Systems Engineering. Studies in Computational Intelligence. 2009. Vol. 259. Springer, Berlin, Heidelberg. P. 177204.

16. Saltan M., Terzi S., Küçüksille E.U. Backcalculation of pavement layer moduli and Poisson's ratio using data mining // Expert Systems With Applications. 2011. Vol. 38, 3. P. 2600-2608.

17. Saric A., Pozder M. Artificial Neural Networks Application in the Backcalculation Process of Flexible Pavement Layers Elasticity Modulus. // In: Hadžikadić M., Avdaković S. (eds) Advanced Technologies, Systems, and Applications II. IAT 2017. Lecture Notes in Networks and Systems. Vol. 28. Springer, Cham. P. 549-559.

18. Tarantola A. Inverse Problem Theory and Methods for Model Parameter Estimation. Philadelphia: SIAM, 2005. Society for Industrial and Applied Mathematics.

19. Tarantola A., Valette B., Generalized nonlinear inverse problems solved using the least-squares criterion // Rev. Geophys. Space Phys., 1982. Vol. 20, No 2. P. 219-232.

20. Tikhonov A.N., Arsenin V.Y. Solutions of III-Posed Problems // John Wiley and Sons. 1977.

21. Zhang Y., Roesler J.R., Huang Z. A method for evaluating CRCP performance based on edge-loaded FWD test. Mater Struct 53, 46 (2020). https://doi.org/10.1617/s11527-020-01481-0. Art. no. 46.

Университет таможенного дела

и фонансов,

Днепр,

Украина

Поступила в редколлегию 26.04.20 